\title{
Optimale Auslegung von getriebelosen, permanentmagneterregten Windgeneratoren mit Zahnspulenwicklung und massivem Rotorjoch
}

\author{
N. ErdD, A. Binder
}

Getriebelose Windgeneratoren mit Zahnspulen und massivem Rotorjoch sowie der Möglichkeit zum Weiterbetrieb bei teilweisem Umrichterausfall stellen eine besondere Herausforderung in der Auslegung dar. Zunächst wird untersucht, welche Zahnspulenwicklungen im Hinblick auf die zulässigen Rotorverluste geeignet sind. Nachdem entsprechende Wicklungsvarianten gefunden sind, wird die Maschinengeometrie optimiert. Dabei wird das Optimierungspotenzial, welches in den kurzen Wickelköpfen der Zahnspulenmaschine liegt, berücksichtigt. Nach der Optimierung erfolgt eine Nachrechnung der Verlustbilanz und eine Auswertung der magnetischen Geräuschanregung mithilfe von transienten, nichtlinearen Finite-Elemente-Simulationen.

Schlüsselwörter: Windgenerator; Auslegung; Optimierung; Zahnspulenwicklung; Wirbelströme

Optimal design of direct drive permanent magnet wind generators with concentrated winding and solid rotor yoke.

Gearless wind generators with concentrated windings, massive rotor yoke and redundancy against inverter failure provide a challenging design task. First of all, suitable windings have to be found regarding the rotor losses due to eddy currents. After finding promising winding topologies, an optimization of the machine geometry is carried out utilizing the benefits of the short winding overhang due to the concentrated winding. Finally, a calculation of the efficiency and magnetic noise excitation is carried out for the chosen optimal machine designs by transient, non-linear finite element simulations.

Keywords: wind generator; design; optimization; concentrated winding; eddy current

Eingegangen am 25. März 2020, angenommen am 27. Mai 2020, online publiziert am 10. Juni 2020

(c) The Author(s) 2020

\section{Einleitung}

In getriebelosen Windenergieanlagen großer Leistung von ca. $1 \mathrm{MW}$... $10 \mathrm{MW}$ werden permanentmagneterregte Synchronmaschinen (PMSM) mit massivem Rotorjoch in Außenläufer-Bauweise eingesetzt $[6,21,31]$. Durch die fehlende Lamellierung des Rotoreisens können dort unter Umständen infolge harmonischer Effekte hohe Wirbelstromverluste auftreten, die einerseits den Wirkungsgrad des Generators herabsetzen und andererseits zu einer unzulässigen Erwärmung der dort eingesetzten Selten-Erd-Magnete (zumeist NdFeB-Material) führen können [20, 26, 28]. Um Rotorwirbelstromverluste wirkungsvoll zu begrenzen, wird die Statorwicklung vorzugsweise als verteilte Wicklung ausgeführt. Diese Wicklungen weisen nur wenige und amplitudenmäßig relativ kleine räumliche Harmonische des Statorfeldes auf, welche asynchron zum Rotor umlaufen und diesen induzieren. Im Folgenden werden zwei Entwicklungstrends vorgestellt, die zu problematischen Harmonischen im Statorfeld führen können:

Erstens werden fallweise PMSM mit Zahnspulenwicklungen eingesetzt. Im Jahr 1996 wurde von Spooner et al. ein modulares Zahnspulensystem für getriebelose PMSM-Windgeneratoren ausgelegt und als Demonstrator im Labormaßstab gebaut [24]. Kommerziell verfügbar sind getriebelose PMSM-Windenergieanlagen mit Zahnspulenwicklungen seit Mitte der 2000er-Jahre beispielsweise beim Unternehmen Leitner [16, 22]. Sowohl die Arbeiten von Mouty [19] als auch von Messoll [18] zeigen das große Potential von Zahn- spulenwicklungen in getriebelosen PMSM-Windenergieanlagen zur weiteren Steigerung der Einheitsleistung bei begrenzter Turmkopfmasse und adressieren damit die aktuellen Herausforderungen bei der Auslegung größter Offshore-Windenergieanlagen.

Zahnspulenwicklungen haben im Gegensatz zu verteilten Wicklungen einen axial sehr kurzen Wickelkopf, der keine Überkreuzungen der Spulen aufweist, so dass diese Wicklungen kostengünstig und robust gefertigt werden können. Ferner werden dadurch axialer Bauraum sowie Stromwärmeverluste im Wickelkopf reduziert. Überdies erlauben Zahnspulenwicklungen relativ einfach eine modulare Statorbauweise, die auf fertig bewickelten und elektrisch isolierten Statorsegmenten beruht und bei großen direktangetriebenen Generatoren mit Außendurchmessern größer als $4 \mathrm{~m}$ bei Straßentransporten zwangsläufig notwendig ist.

Zweitens speisen hochpolige Generatoren fallweise über mehrere dreiphasige Umrichter in das Netz ein. Hierbei wird ein fehlertoleranter Weiterbetrieb (Redundanzbetrieb) gegenüber einer Störung eines einzelnen Umrichters erreicht, was nach [23] einen im Vergleich zu anderen Ursachen relativ häufiger Grund für den Ausfall

Erd, Nicolas, Institut für Elektrische Energiewandlung, Technische Universität Darmstadt, Landgraf-Georg-Str. 4, 64283 Darmstadt, Deutschland

(E-Mail: nerd@ew.tu-darmstadt.de); Binder, Andreas, Institut für Elektrische Energiewandlung, Technische Universität Darmstadt, Landgraf-Georg-Str. 4, 64283 Darmstadt, Deutschland (E-Mail: abinder@ew.tu-darmstadt.de) 


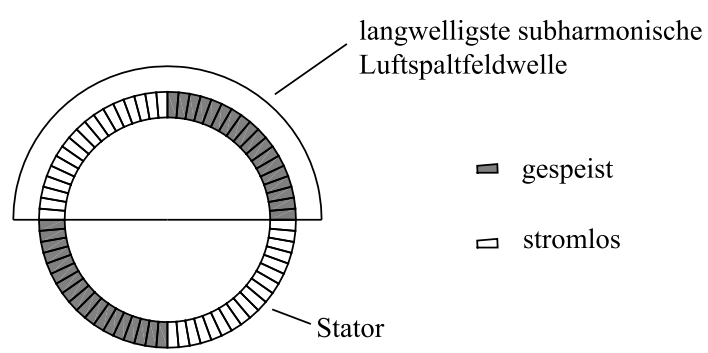

Abb. 1. Vier-Quadranten-Speisung im Redundanzbetrieb. Ein Umrichter speist zwei gegenüberliegende Quadranten. Die anderen beiden Quadranten sind wegen des defekten zweiten Umrichters stromlos

einer Windenergieanlage ist. Im Fehlerfall eines Umrichters werden die betroffenen Zweige der Wicklung vom fehlerhaften Umrichter freigeschaltet und der Betrieb unter Verwendung des noch funktionsfähigen Umrichters mit reduzierter Leistung fortgesetzt. In diesem Redundanzbetrieb wird die Statorwicklung, wie in Abb. 1 dargestellt, abschnittsweise bestromt. Dabei treten zusätzliche langwellige Subharmonische im Statorfeld auf, die aufgrund ihrer Langwelligkeit den magnetischen Luftspalt mit vergleichsweise hoher Amplitude überwinden und hohe Wirbelströme auf der Rotorseite verursachen können.

Im vorliegenden Beitrag wird die optimale Generatorauslegung unter der Maßgabe des Einsatzes von Zahnspulenwicklungen und der redundanten Speisung mit zwei SpannungszwischenkreisUmrichtern behandelt. Das wesentliche Optimierungspotential der Zahnspulenwicklungen gegenüber verteilten Wicklungen liegt in den axial kürzeren Wickelköpfen, so dass bei gleichen Außenabmessungen durch die größere axiale Eisenlänge ein größeres Aktivvolumen erreicht wird. Dadurch wird bei gleicher Leistung eine geringere elektromagnetische Ausnutzung möglich, so dass ggf. der Wirkungsgrad steigt.

Hierfür werden zunächst geeignete Zahnspulenwicklungen identifiziert. Dies geschieht durch eine schnelle, analytische Abschätzung der Rotorwirbelstromverluste. Danach erfolgt eine optimale Maschinenauslegung verschiedener Zahnspulenvarianten durch den Einsatz von genetischen Optimierungs-Algorithmen [2, 5]. Aus den gewonnenen Pareto-Fronten werden Maschinenentwürfe mit gleicher Magnetmasse ausgewählt und mit Hilfe transienter, nichtlinearer Finite-
Elemente-Berechnungen nachgerechnet, um die Entwurfsvarianten anhand ihrer Gesamtverluste und des Grundschwingungsleistungsfaktors vergleichen zu können. Abschließend findet eine rechnerische Untersuchung zur Geräuschanregung statt.

\section{Zahnspulenwicklungen und Wirbelstromverluste im massiven Rotorjoch}

Die Vielfalt an möglichen Zahnspulenwicklungen macht es notwendig, eine Vorauswahl von möglichst wenigen sinnvollen Varianten zu treffen, für die im Anschluss eine rechenintensive Optimierung des Blechschnitts durchgeführt wird.

Zahnspulenwicklungen können durch Strangzahl $m$, Lagenzahl, Lochzahl $q$ und Polpaarzahl $p$ beschrieben werden [3]. In der vorliegenden Auslegung sind vor allem die Wirbelstromverluste im massiven Rotorjoch kritisch, sodass eine Zahnspulenwicklung mit möglichst wenigen und amplitudenmäßig geringen räumlichen Harmonischen des Statorfeldes gewählt werden muss. ZweischichtZahnspulenwicklungen weisen generell geringere Harmonische auf als Einschicht-Zahnspulenwicklungen [6]. Deshalb werden letztere für die Auslegung nicht beachtet. Die Lochzahl einer Zahnspulenwicklung kann als echter Bruch mit $q=Z / N$ ausgedrückt werden, wobei $Z$ und $N$ natürliche, teilerfremde Zahlen sind. Zahnspulen mit akzeptablem Wicklungsfaktor nahe Eins liegen im Bereich $q \approx 1 / m$, aber $q \neq 1 / m$, da $q=1 / m$ auf unsymmetrische Wicklungen führt und ausgeschlossen wird [3]. Mit steigendem Bruchlochnenner $N$ nimmt die Anzahl der Nuten je Urwickelschema zu, und die Ordnungszahl der Arbeitswelle steigt. Das bedeutet, dass mit steigendem Bruchlochnenner $N$ immer mehr Unterwellen hinzukommen, die aufgrund ihrer Langwelligkeit den magnetisch wirksamen Luftspalt (als Summe aus Luftspaltweite und Magnethöhe) relativ leicht überwinden und hohe Wirbelströme im massiven Rotorjoch treiben können. Daher kommen für die hier betrachtete Anwendung nur Bruchlochwicklungen mit relativ kleinem Bruchlochnenner $N$ in Frage. Der Sonderfall der Zweischicht-Bruchlochwicklung mit $q=1 / 2$ weist keine Unterwellen auf und erscheint daher in besonderem MaBe geeignet. Allerdings beträgt der Wicklungsfaktor der Arbeitswelle $k_{\mathrm{w}, p}$ in diesem Fall nur 0.866 und ist im Vergleich zum Optimalwert Eins relativ gering.

Im Folgenden werden die in Tab. 1 angegebenen vier Zahnspulenwicklungen und eine verteilte Wicklung als Kandidaten betrachtet: Drei Zahnspulenwicklungen mit $m=3$, die im Redundanzbetrieb mit abschnittsweiser Speisung ganzer Urwickelschemen weiter

Tab. 1. Gewählte Wicklungsvarianten für die Voruntersuchung. Z: Zahnspule, V: Verteilte Wicklung, *: analytische Berechnung

\begin{tabular}{|c|c|c|c|c|c|}
\hline Wicklungsparameter & Z1 & $\mathrm{Z2}$ & $\mathrm{Z3}$ & Z4 & $\mathrm{V} 1$ \\
\hline Strangzahl $m$ & 3 & 3 & 3 & 6 & 3 \\
\hline Lagenzahl & 2 & 2 & 2 & 2 & 1 \\
\hline Lochzahl q & $1 / 2$ & $2 / 5$ & $3 / 8$ & $1 / 5$ & 1 \\
\hline Polpaarzahl im Urschema $p_{u}$ & 1 & 5 & 4 & 5 & 1 \\
\hline Ordnungszahlen der Unterwellen & - & 1 & $1,-2$ & - & - \\
\hline Nutzahl im Urschema $Q_{u}$ & 3 & 12 & 9 & 12 & 6 \\
\hline Wicklungsfaktor der Arbeitswelle $k_{\mathrm{w}, p}$ & 0.866 & 0.933 & 0.945 & 0.966 & 1 \\
\hline Ober- und Unterwellenstreuziffer $\sigma_{\mathrm{o}}$ & 0.462 & 0.968 & 1.18 & 0.836 & 0.097 \\
\hline Polzahl $2 p$ & 192 & 180 & 192 & 180 & 144 \\
\hline Nutzahl Q & 288 & 216 & 216 & 216 & 432 \\
\hline Wirbelstromverluste im Rotorjoch im Normalbetrieb* & $28.1 \mathrm{~kW}$ & $239 \mathrm{~kW}$ & $307 \mathrm{~kW}$ & $160 \mathrm{~kW}$ & $0.6 \mathrm{~kW}$ \\
\hline Wirbelstromverluste im Rotorjoch im Redundanzbetrieb* & $34.6 \mathrm{~kW}$ & 146 kW & $187 \mathrm{~kW}$ & $318 \mathrm{~kW}$ & $23.1 \mathrm{~kW}$ \\
\hline Rotorverluste thermisch zulässig & ja & ja & nein & nein & ja \\
\hline
\end{tabular}


Tab. 2. Vorgaben zur Abschätzung der Wirbelstromverluste im Rotorjoch

\begin{tabular}{|c|c|}
\hline Bezeichnung & Wert \\
\hline $\begin{array}{l}\text { Statoraußendurchmesser } d_{\mathrm{s}, 0}=2 R_{1} \\
\text { (Außenläufer) }\end{array}$ & $6355 \mathrm{~mm}$ \\
\hline $\begin{array}{l}\text { Ideelle Eisenlänge } l_{\mathrm{e}} \\
\text { Magnetisch wirksame Luftspaltweite } \\
b=R_{2}-R_{1} \text { (Luftspalt und } \\
\text { Magnethöhe) }\end{array}$ & $\begin{array}{l}1635 \mathrm{~mm} \\
30 \mathrm{~mm}\end{array}$ \\
\hline $\begin{array}{l}\text { Rotorjochinnendurchmesser } \\
d_{r, i}=2 R_{2}\end{array}$ & $6415 \mathrm{~mm}$ \\
\hline $\begin{array}{l}\text { Rotorjochaußendurchmesser } \\
d_{r, 0}=2 R_{3}\end{array}$ & $6515 \mathrm{~mm}$ \\
\hline $\begin{array}{l}\text { Bemessungsdrehzahl } n_{N} \\
\text { Bemessungsdrehmoment } M_{N} \\
\text { Effektivwert der } \\
\text { Strombelagsarbeitswelle }\left|\underline{K}_{p}\right| / \sqrt{2}\end{array}$ & $\begin{array}{l}10.2 \mathrm{~min}^{-1} \\
8.3 \mathrm{MNm} \\
1500 \mathrm{~A} / \mathrm{cm}\end{array}$ \\
\hline $\begin{array}{l}\text { Rel. magnetische Permeabilität des } \\
\text { Rotorjochs } \mu_{r, \text { rot }}\end{array}$ & 100 \\
\hline $\begin{array}{l}\text { Elektrische Leitfähigkeit des } \\
\text { Rotorjochs } \sigma_{\text {rot }}\end{array}$ & $6.29 \mathrm{MS} / \mathrm{m}$ \\
\hline $\begin{array}{l}\text { Wärmeübergangskoeffizient } \alpha \text { bei } \\
F_{\text {rot }}\end{array}$ & $125 \mathrm{~W} /\left(\mathrm{m}^{2} \mathrm{~K}\right)$ \\
\hline $\begin{array}{l}\text { Gesamte wirksame Rotorkühlfläche: } \\
\text { Mantelfläche des Rotorjochs (innen } \\
\text { und außen) } F_{\text {rot }}\end{array}$ & $66.4 \mathrm{~m}^{2}$ \\
\hline Zulässige Rotorerwärmung $\Delta \vartheta_{\text {rot }}$ & $30 \mathrm{~K}$ \\
\hline Zulässige Wirbelstromverluste $P_{\text {rot }}$ & $249 \mathrm{~kW}$ \\
\hline
\end{tabular}

arbeiten können, und eine Zahnspulenwicklung mit $m=6$, die im Redundanzbetrieb nur eine teilweise Speisung des Urwickelschemas mit dem verbleibenden intakten dreiphasigen Umrichter ermöglicht. Zu Vergleichszwecken wird auch eine verteilte Einschichtwicklung mit $q=1$ betrachtet. Die Polzahlen der abgeleiteten Maschinenvarianten wurden wie folgt gewählt: Ausgehend von 180 Polen für $q=2 / 5$ sollen sich ähnliche Spulenbreiten ergeben, d.h. die Zahnspulenmaschinen werden mit deutlich mehr Polen ausgeführt als die Maschine mit der verteilten Wicklung. Überdies muss der vorteilhafte Redundanzbetrieb mit zwei gegenüberliegenden, gespeisten Quadranten in ganzen Urwickelschemen möglich sein (s. Abb. 1). Diese Topologie bei abschnittsweiser Statorspeisung ist besonders vorteilhaft, da einerseits die am Umfang ungleichmäßig wirkenden Radial- und Schubkräfte sich durch die diametral gegenüberliegenden gespeisten und ungespeisten Abschnitte derart kompensieren, dass keine resultierenden translativen Kräfte auf die Hauptelemente wirken. Andererseits wurde in [7] gezeigt, dass diese Speisung durch die möglichst großen Speisegruppen vergleichsweise geringe zusätzliche Rotorwirbelstromverluste bei abschnittsweiser Speisung erzeugt.

Die Varianten werden anhand der analytisch berechneten Rotorjochwirbelstromverluste im Normalbetrieb und "Redundanzbetrieb" (Notbetrieb) bei Ausfall eines Umrichters bewertet. Hierzu werden die in Tab. 2 dargestellten Abmessungen und der dargestellte Strombelag der Arbeitswelle $K_{p}$ angenommen. Des Weiteren wird im Rahmen dieser analytischen Berechnung nur die Stromgrundschwingung betrachtet und zusätzliche Rotorverluste durch Stromoberschwingungen werden nicht berücksichtigt. Über die Vorgabe der zulässigen Rotorerwärmung ergibt sich unter Berücksichtigung des

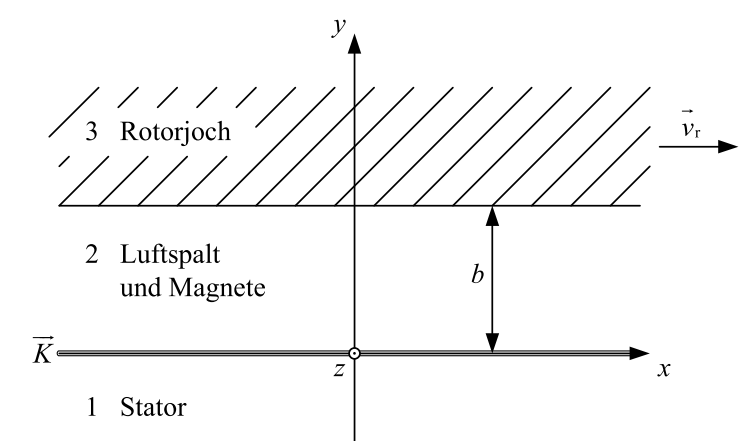

Abb. 2. Analytisches, kartesisches Modell zur Berechnung der Wirbelströme im Rotorjoch ( $\vec{v}_{r}$ : Rotorumfangsgeschwindigkeit, $\vec{K}$ : Statorstrombelag)

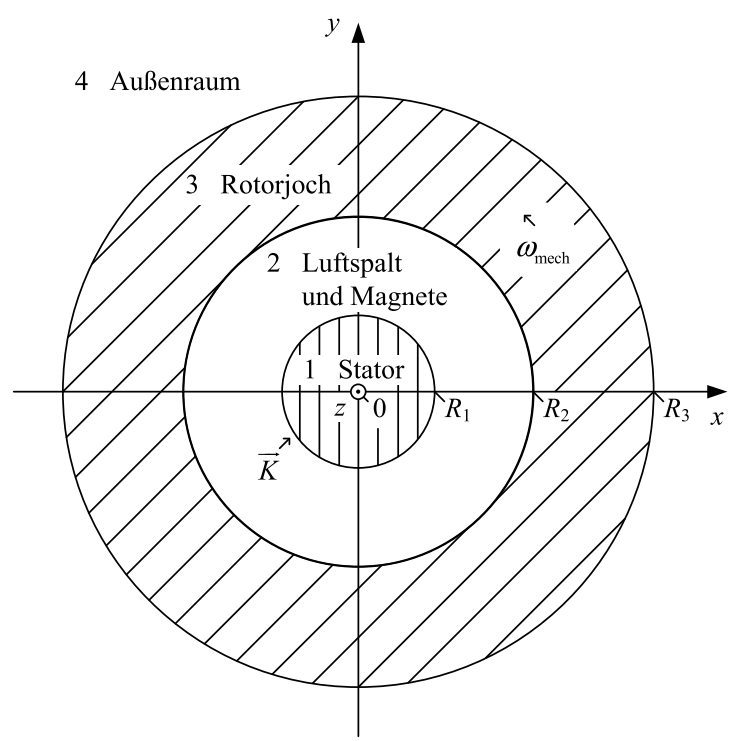

Abb. 3. Analytisches, zylindrisches Modell zur Berechnung der Wirbelströme im Rotorjoch $\left(\omega_{\text {mech }}\right.$ : mech. Winkelgeschwindigkeit des Rotors, $\vec{K}$ : Statorstrombelag)

inneren Luft-Kühlkreislaufs und der äußeren konvektiven Kühlung des Rotors eine obere Grenze für die zulässigen Rotorverluste in Höhe von 249 kW. Damit liegt die thermische Grenze der Rotorverluste bei ca. $2.8 \%$ der Eingangsleistung. Dieser vergleichsweise hohe Wert ist nur aufgrund der Außenläuferanordnung mit beidseitiger Rotorkühlung möglich.

\subsection{Analytische Wirbelstromberechnung}

Die analytische Berechnung der Wirbelströme erfolgt für zweidimensionale Ersatzanordnungen mit der Annahme magnetisch und elektrisch linearer Medien und für eine zeitharmonische Anregung durch den Statorstrombelag $\vec{K}$. Die Wirkung eines Wellenspektrums kann in diesem Fall durch Superposition der Teilwellen ermittelt werden.

Während in $[8,10,11,15,25]$ die Lösung des Wirbelstromproblems in kartesischen Koordinaten für die abgewickelte Maschine dargestellt ist (s. Abb. 2), wird hier auf die Berechnung in Zylinderkoordinaten mit Berücksichtigung der Krümmung der Maschine nach $[1,4]$ eingegangen (s. Abb. 3). Die Maschine wird durch konzentrische Regionen bei Vernachlässigung der Nutung modelliert. Mit der 
Annahme einer hinreichend feinen Magnetsegmentierung sind die Wirbelströme in den Magneten vernachlässigbar klein, sodass die elektrische Leitfähigkeit der Magnete zu Null gesetzt wird. Da zusätzlich die relative Permeabilität der Selten-Erd-Permanentmagnete nahezu Eins ist und hier zu $\mu_{r \text {,mag }}=1$ gesetzt wird, können Luftspalt und Magnete als eine gemeinsam magnetisch wirksame Region modelliert werden (s. Region 2 in Abb. 3).

Der anregende Statorstrombelag $\vec{K}$ wird in (1) als $\vec{K}_{\mathrm{s}}$ in einem statorfesten Koordinatensystem $\left(r_{s}, \theta_{s}, z\right)$ (Index $s$ ) als Superposition von Statorstrombelagswellen verschiedener absoluter räumlicher Ordnungszahlen $v$ aber gleicher Statorkreisfrequenz $\omega_{\mathrm{s}}$ beschrieben. In (3) wird die Amplitude des Strombelags der räumlichen Ordnung $v$ als komplexe Zahl $\underline{K}_{v}$ eingeführt.

$$
\begin{aligned}
& \vec{K}_{s}\left(\theta_{s}, t\right)=\sum_{v} \vec{K}_{s, v}\left(\theta_{s}, t\right) \\
& \vec{K}_{s, v}\left(\theta_{s}, t\right)=K_{s, v}\left(\theta_{s}, t\right) \cdot \vec{e}_{z} \\
& K_{s, v}\left(\theta_{s}, t\right)=\operatorname{Re}\left\{\underline{K}_{v} \cdot \mathrm{e}^{-\mathrm{j} v \theta_{s}} \cdot \mathrm{e}^{\mathrm{j} \omega_{s} t}\right\}
\end{aligned}
$$

Da die Lösung des Wirbelstromproblems in einem mit der Rotorkreisfrequenz $\omega_{\text {mech }}$ mitrotierendem Koordinatensystem erfolgt, wird zunächst der Statorstrombelag $\vec{K}$ in diesem rotorfesten Koordinatensystem $\left(r_{r}, \theta_{r}, z\right)$ als $\vec{K}_{r}$ (Index $\left.r\right)$ beschrieben. Es wird der Spezialfall betrachtet, dass die Rotordrehzahl der Synchrondrehzahl, wie in (4) dargestellt, entspricht. Ziel ist die Beschreibung der Statorstrombelagswelle in Rotorkoordinaten in der Form (6). Beim Übergang in das rotorfeste Koordinatensystem bleiben die räumlichen Ordnungszahlen $v$ der betrachteten Statorstrombelagswellen erhalten, aber die Kreisfrequenzen im Rotorsystem $\omega_{\mathrm{r}, v}$ hängen wegen der Rotordrehbewegung von $v$ nach (7) ab.

$$
\begin{aligned}
& \omega_{\text {mech }}=\frac{\omega_{\mathrm{s}}}{p} \\
& \vec{K}_{r, v}\left(\theta_{\mathrm{r}}, t\right)=K_{\mathrm{r}, v}\left(\theta_{\mathrm{r}}, t\right) \cdot \vec{e}_{z} \\
& K_{\mathrm{r}, v}\left(\theta_{\mathrm{r}}, t\right)=\operatorname{Re}\left\{\underline{K}_{v} \cdot \mathrm{e}^{-\mathrm{j} v \theta_{\mathrm{r}}} \cdot \mathrm{e}^{\mathrm{j} \omega_{r, v} t}\right\} \\
& \omega_{\mathrm{r}, v}=\left(1-\frac{v}{p}\right) \cdot \omega_{\mathrm{s}}
\end{aligned}
$$

Die Lösung des Feldproblems erfolgt mit Hilfe des magnetischen Vektorpotentials $\vec{A}$ nach (8) mit der Coulomb-Eichung nach (9). Die Maxwell'schen Gleichungen führen für leitfähige Regionen auf die Diffusionsgleichung (10).

$$
\begin{aligned}
& \vec{B}=\nabla \times \vec{A} \\
& \nabla \cdot \vec{A}=0 \\
& \nabla^{2} \vec{A}=\mu \sigma \frac{\partial}{\partial t} \vec{A}
\end{aligned}
$$

Entsprechend der Anregung durch die Statorstrombelagswellen wird das magnetische Vektorpotential in rotorfesten Koordinaten (Index $r$ ) für jede Kreisregion $i=1 \ldots 4$ und jede anregende Strombelagsordnung $v$ getrennt nach dem Superpositionsprinzip gelöst, s. (11). Aus der Diffusionsgleichung (10) wird für zeitharmonische Anregungen und leitfähige Regionen die Helmholtz-Gleichung (12) unter Einführung des Parameters $\underline{\kappa}_{i, v}$ nach (13). In nicht-leitfähigen Regionen wird zur Laplace-Gleichung (14) vereinfacht.

$$
\begin{aligned}
& \vec{A}_{r, i, v}\left(r_{r}, \theta_{r}, t\right)=\operatorname{Re}\left\{\vec{A}_{r, i, v}\left(r_{r}, \theta_{r}\right) \cdot \mathrm{e}^{\mathrm{j} \omega_{r, v} t}\right\} \\
& \nabla^{2} \vec{A}_{r, i, v}\left(r_{r}, \theta_{r}\right)=\underline{\kappa}_{i, v}^{2} \cdot \underline{\vec{A}}_{r, i, v}\left(r_{r}, \theta_{r}\right) \quad \text { für: } \sigma_{i} \neq 0 \\
& \underline{\kappa}_{i, v}^{2}=j \omega_{r, v} \mu_{i} \sigma_{i}
\end{aligned}
$$

$$
\nabla^{2} \underline{A}_{r, i, v}\left(r_{r}, \theta_{r}\right)=0 \quad \text { für: } \sigma_{i}=0
$$

Der anregende Statorstrombelag weist ausschließlich eine $z-$ Komponente auf, daher gilt dies auch für das Vektorpotential (15). Mit Hilfe des Separationsansatzes nach (16) werden die partiellen Differentialgleichungen (12) bzw. (14) zu gewöhnlichen Differentialgleichungen umgeformt.

$$
\begin{aligned}
& \vec{A}_{r, i, v}\left(r_{r}, \theta_{r}\right)=\underline{A}_{r, i, v}\left(r_{r}, \theta_{r}\right) \cdot \vec{e}_{z} \\
& \underline{A}_{r, i, v}\left(r_{r}, \theta_{r}\right)=\underline{R}_{i, v}\left(r_{r}\right) \cdot \mathrm{e}^{-\mathrm{j} v \theta_{r}} \cdot \mathrm{e}^{\mathrm{j} \omega_{r, v} t}
\end{aligned}
$$

Im Folgenden werden die drei Indizes $r, i$ und $v$ zur besseren Übersicht weggelassen, obwohl weiterhin die Lösung in Rotorkoordinaten, getrennt für jede Region und anregende räumliche Ordnung, erfolgt.

\subsubsection{Lösung der Helmholtz-Gleichung}

Um die Funktion $\underline{R}(r)$ im Fall der leitfähigen Region $i=3$ in Abb. 3 zu ermitteln, ist der Laplace-Operator in Zylinderkoordinaten nach (17) in die Helmholtz-Gleichung (12) einzusetzen. Unter Anwendung des Separationsansatzes (16) vereinfacht sich das Problem zur gewöhnlichen Differentialgleichung (18) als modifizierte BesselDifferenzialgleichung. Diese wird durch eine Superposition der modifizierten Bessel-Funktion 1. Art $\underline{I}_{v}(\underline{\kappa} r)$ und der modifizierten BesselFunktion 2. Art $\underline{J}_{\nu}(\underline{\kappa} r)$ jeweils mit der räumlichen Ordnung $v$ gelöst. Die Skalierung des Arguments der modifizierten Bessel-Funktionen mit $\underline{\kappa}$ gemäß (19) führt zu komplexwertigen Funktionen.

$$
\begin{aligned}
& \nabla^{2} \underline{\vec{A}}=\frac{1}{r} \frac{\partial}{\partial r}\left(r \frac{\partial \underline{A}}{\partial r}\right)+\frac{1}{r^{2}} \frac{\partial^{2} \underline{A}}{\partial \theta^{2}} \\
& r^{2} \underline{R}^{\prime \prime}(r)+r \underline{R}^{\prime}(r)-\left((\underline{\kappa} r)^{2}+v^{2}\right) \underline{R}(r)=0 \\
& \underline{R}(r)=\underline{C} \cdot \underline{I}_{\nu}(\underline{\kappa} r)+\underline{D} \cdot \underline{J}_{\nu}(\underline{\kappa} r)
\end{aligned}
$$

\subsubsection{Lösung der Laplace-Gleichung}

Für die nicht-leitfähigen Regionen $i=1,2$ und 4 in Abb. 3 ergibt der Separationsansatz (16) durch Einsetzen von (17) in die LaplaceGleichung (14) die gewöhnliche Euler'sche Differentialgleichung (20) mit der allgemeinen Lösung nach (21).

$$
\begin{aligned}
& r^{2} \underline{R}^{\prime \prime}(r)+r \underline{R}^{\prime}(r)-v^{2} \underline{R}(r)=0 \\
& \underline{R}(r)=\frac{\underline{C}}{r^{v}}+\underline{D} \cdot r^{\nu}
\end{aligned}
$$

Über die Rand- und Stetigkeitsbedingungen werden für jede Region $i=1 \ldots 4$ die freien Koeffizienten $\underline{C}$ und $\underline{D}$ bestimmt. Dabei ist die Normalkomponente der magnetischen Flussdichte $\underline{B}_{r}$ an den Grenzflächen zwischen den Regionen stetig. Die Tangentialkomponente der magnetischen Feldstärke $\underline{H}_{\theta}$ springt am Übergang der benachbarten Regionen 1 und 2 um den eingeprägten Strombelag $\underline{K}$ an der Grenzschicht und ist sonst stetig. Durch diese Randbedingungen wird ein lineares Gleichungssystem für alle acht Konstanten $\underline{C}_{i}, \underline{D}_{i}$ mit $i=1 \ldots 4$ aufgestellt, um diese zu berechnen. Alternativ werden die Feldgrößen $\underline{B}_{r}$ und $\underline{H}_{\theta}$ an den Grenzflächen sukzessive von innen nach außen mit Hilfe sogenannter Übertragungsmatrizen berechnet [1].

\subsubsection{Berechnung der Wirbelstromverluste im Rotorjoch}

Nach der Berechnung des Vektorpotentials erfolgt die Bestimmung der Wirbelstromverluste über den Poynting'schen Satz [32]. Es werden für eine Zylinderhüllfläche im Luftspalt $F_{\delta}$ die komplexen Amplituden für die $z$-Komponente des elektrischen Feldes $\underline{E}_{\delta, z, v}$ und die $\theta$-Komponente der magnetischen Feldstärke $\underline{H}_{\delta, \theta, v}$ in Region 2 
ermittelt. Die spektralen Rotorverluste $P_{\text {rot, } v}$ ergeben sich dann aus (22) durch Realteilbildung.

$$
P_{\mathrm{rot}, v}=\frac{1}{2} \cdot F_{\delta} \cdot \operatorname{Re}\left\{\underline{E}_{\delta, z, v} \cdot \underline{H}_{\delta, \theta, v}^{*}\right\}
$$

\subsection{Spektrale Verlustverteilung für die Wicklungvarianten} Für die Darstellung der spektralen Zusammensetzung der in Tab. 1 aufgeführten kumulierten Rotorjochwirbelstromverluste $P_{\text {rot }}$ werden alle Wicklungsvarianten aus Tab. 1 bei Normalbetrieb und abschnittsweiser Quadranten-Speisung im „Redundanzbetrieb" untersucht. Für die analytische Berechnung des Feldproblems wird das Statorblechpaket als nicht-leitfähige, unendlich permeable Region 1 aufgefasst und die in Tab. 2 aufgeführten Angaben zugrunde gelegt. Insbesondere wird die Bestromung für jede der fünf Wicklungen $\mathrm{Z} 1$ bis $\mathrm{Z} 4$ und $\mathrm{V} 1$ so eingestellt, dass der Effektivwert der Strombelagsarbeitswelle $\left|K_{p}\right| / \sqrt{2}=1500 \mathrm{~A} / \mathrm{cm}$ beträgt, um näherungsweise gleiche Drehmomente für die fünf verschiedenen Varianten zu erreichen. Die Amplituden der Statorfeldharmonischen folgen entsprechend aus den Wicklungsfaktoren $k_{\mathrm{w}, v}$ der jeweiligen Wicklungsvariante. Problematisch ist die Festlegung einer konstanten magnetischen Permeabilität des Rotorjochs $\mu_{r}$,rot, da durch den Skineffekt eine Feldverdrängung des magnetischen Flusses der asynchron umlaufenden Feldwellen in der Rotoroberfläche auftritt. Dies führt zu einer lokalen Eisensättigung in einer Randschicht nahe der Oberfläche, wo die magnetische Flussdichte am größten ist. Die Abschätzung der relativen Permeabilität in Tab. $2 \mathrm{zu} \mu_{\mathrm{r}, \text { rot }}=100$ für diese Sättigung geht auf Vergleiche mit feldnumerischen Simulationen zurück [7].

Die Diskussion der spektralen Verlustverteilungen beginnt mit einer detaillierten Betrachtung von Wicklungsvariante Z2 $(q=2 / 5$, $2 p=180$ ). In Abb. 4 werden untereinander die Wicklungsfaktoren $k_{w, v}$, die Amplituden der anregenden Statorstrombelagswellen als fiktive Feldwellen $B_{f i k, v}$, der Schlupf $\omega_{\mathrm{r}, v} / \omega_{\mathrm{s}}$, die Wellenlänge $\lambda_{v} / \lambda_{p}$ und die kumulierten Rotorjochwirbelstromverluste $\sum P_{\text {rot, }, v}$ über der räumlichen Wellenordnung $v / p$ gezeigt. Die fiktiven Feldwellenamplituden $B_{\mathrm{fik}, v}$ dienen der Darstellung der anregenden Strombelagswellen und berechnen sich nach (23). Sie entsprechen der Radialkomponente der resultierenden Statorfeldwellen ohne Wirbelströme und ideal permeablem Eisen in Stator und Rotor bei eindimensionaler Berechnung des Luftspaltfelds.

$$
B_{\mathrm{fik}, v}=\frac{\mu_{0}}{b} \cdot \frac{R_{1}+b / 2}{v} \cdot\left|\underline{K}_{v}\right|
$$

Die kumulierten Rotorjochwirbelstromverluste sind in den Abb. 4 bis 13 sowohl für das zylindrische als auch das kartesische Modell angegeben und weisen aufgrund des großen Maschinendurchmessers und der hohen Polzahl nur marginale Abweichungen $(<2 \%)$ auf.

Für die Wicklungvariante Z2 werden die Verluste maßgeblich durch die zur Arbeitswelle benachbarte Feldoberwelle $(\nu / p=-1.4)$ und die eine auftretende Feldunterwelle $(v / p=-0.2)$ gebildet. Die benachbarte Feldoberwelle weist bei Zahnspulenwicklungen ungünstigerweise denselben Wicklungsfaktor auf wie die Arbeitswelle. Da der spektrale Abstand mit $|v / p|-1=0.4$ relativ gering ausfällt, resultieren sowohl eine hohe Feldamplitude $B_{\text {fik, }}$ als auch ein aufgrund einer ziemlich großen Wellenlänge relativ hohes Bestreben zur Überbrückung des magnetischen Luftspaltes bis zum Rotorjoch. Die Feldunterwelle weist einen relativ geringen Wicklungsfaktor von 0.067 auf. Allerdings wird durch die niedrige Ordnungszahl (vgl. (23)) bzw. die große Wellenlänge von $5 \lambda_{p}$ eine beachtliche Feldamplitude $B_{\mathrm{fik}, v}$ von $36 \%$ der Arbeitswelle gebildet und damit der magnetische Luftspalt mit hoher Amplitude überwunden. Die Oberwellen höherer Ordnung weisen aufgrund der mit $1 /|v|$ fallenden Wellenlänge auch fallende Feldamplituden $B_{\mathrm{fik}, v}$ auf. Durch den
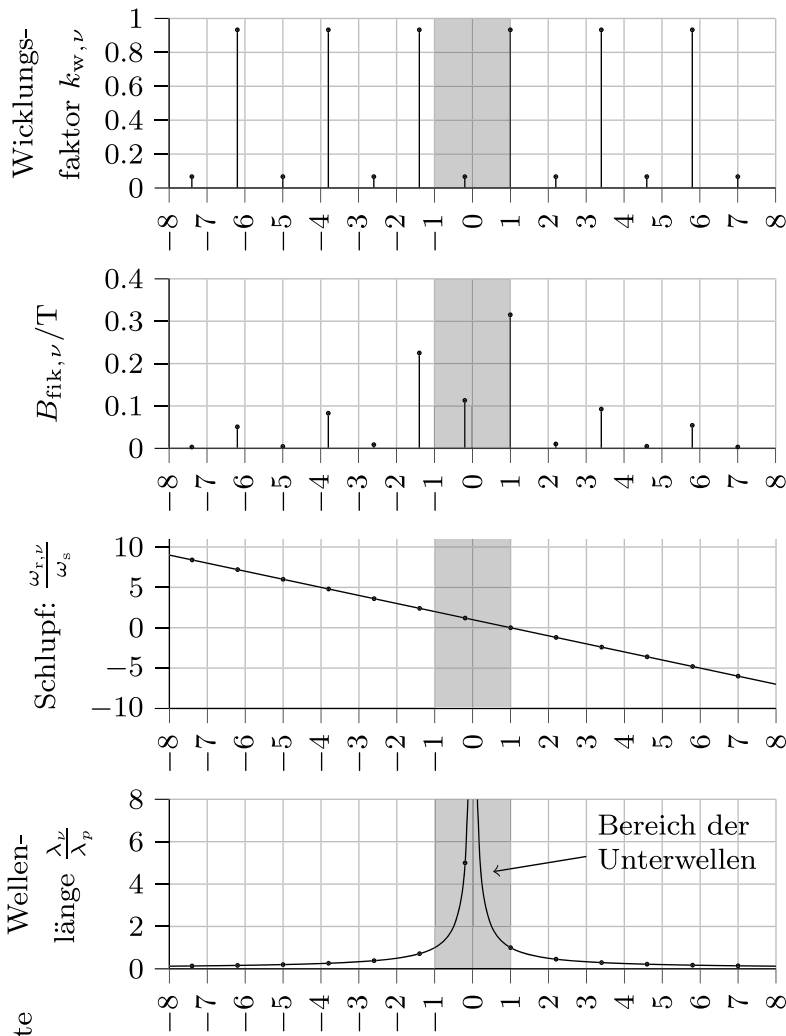

落

$\begin{array}{llllll}1 & 1 & 1 & 1 \\ 0 & 1 & 1 & 1\end{array}$

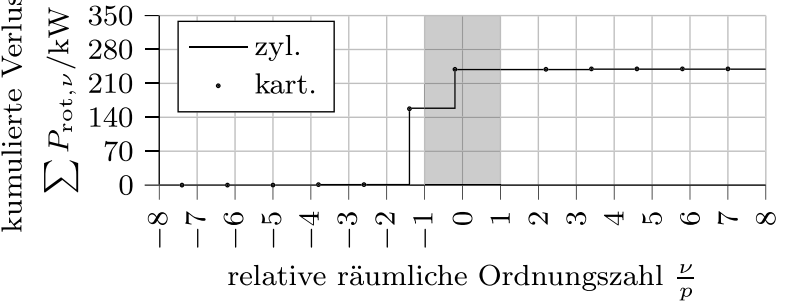

Abb. 4. Statorwicklungsvariante $Z 2(q=2 / 5,2 p=180)$, Normalbetrieb gemäß Tab. 2: Analytische Berechnung der Wirbelstromverluste im Rotorjoch

relativ großen magnetischen Luftspalt von $b=30 \mathrm{~mm}$ erreichen sie nur noch mit sehr geringen Feldamplituden das Rotorjoch und sind für die Gesamtwirbelstromverluste vernachlässigbar.

Die spektrale Darstellung der Berechnungsergebnisse bei abschnittsweiser Speisung der Statorquadranten im Redundanzbetrieb befinden sich für Wicklungsvariante Z2 in Abb. 5. Durch die abschnittsweise Speisung treten zahlreiche zusätzliche Feldober- und Feldunterwellen auf, insbesondere sehr langwellige Unterwellen. Die größte Wellenlänge beträgt den halben Maschinenumfang, bestehend aus einem gespeisten und einem ungespeisten Quadranten. Die Berechnung der resultierenden Wicklungsfaktoren ist in [8] dargestellt und basiert in der numerischen Implementierung auf [30]. Der spektrale Verlauf der Wicklungsfaktoren in Abb. 5 weist gegenüber der vollständig gespeisten Maschine (vgl. Abb. 4) Seitenbänder um die üblichen Harmonischen auf. Dieser Effekt wird in [8] als Faltung des Originalspektrums mit dem Spektrum einer Ausblendfunktion aufgefasst. Da nur zwei von vier Quadranten bestromt werden und die Maschine daher näherungsweise nur das halbe Drehmoment liefert, sind die Amplituden der Statorfeldwellen halbiert. Die kumulierten Rotorwirbelstromverluste fallen mit $146 \mathrm{~kW}$ deutlich geringer aus als bei vollständiger Speisung mit 239 kW. 


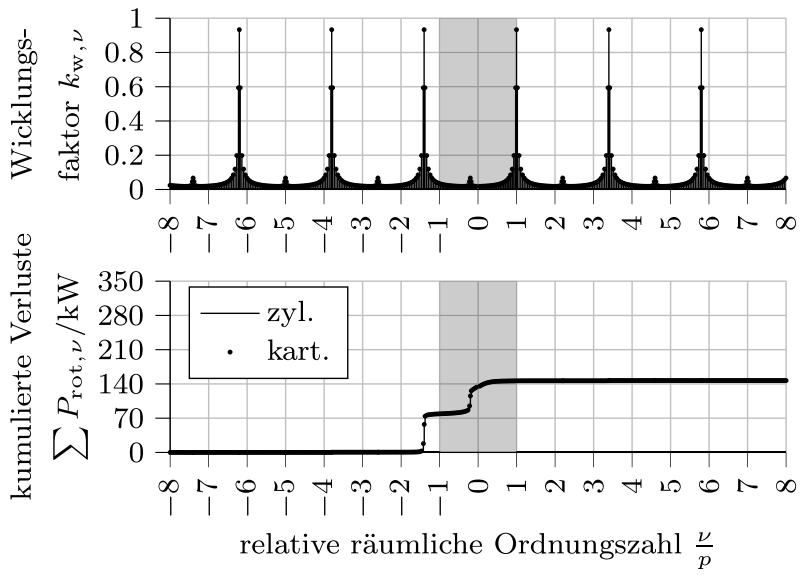

Abb. 5. Statorwicklungsvariante $\mathrm{Z2}(q=2 / 5,2 p=180)$, Redundanzbetrieb mit Quadranten-Speisung gemäß Abb. 1: Analytische Berechnung der Wirbelstromverluste im Rotorjoch

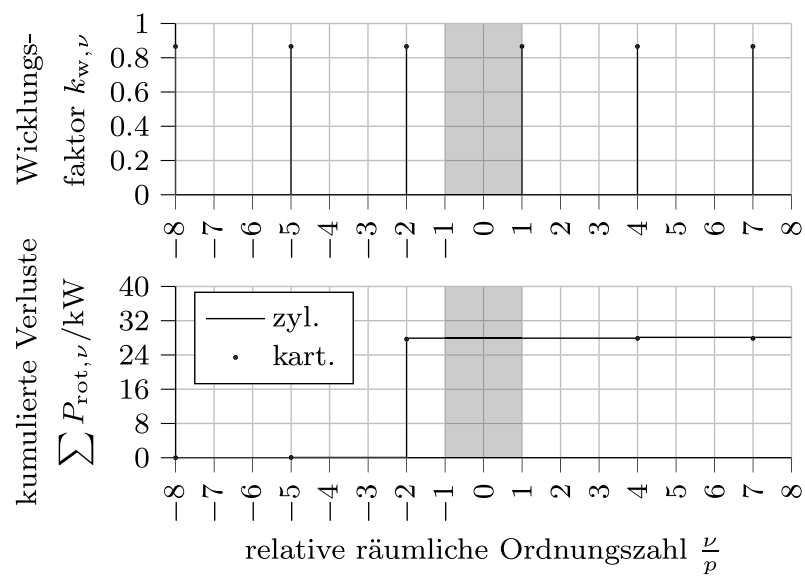

Abb. 6. Wicklungsvariante $Z 1(q=1 / 2,2 p=192)$, Normalbetrieb gemäß Tab. 2: Analytische Berechnung der Wirbelstromverluste im Rotorjoch

Für die Wicklungsvariante Z1 ( $q=1 / 2,2 p=192)$ werden die Rotorjochwirbelstromverluste bei Normalbetrieb in Abb. 6 und für die Quadranten-Speisung im Redundanzbetrieb in Abb. 7 dargestellt. Diese Wicklungsvariante weist im Normalbetrieb keine Unterwelle auf, dafür aber einen relativ kleinen Arbeitswellenwicklungsfaktor von 0.866. Die Verluste werden bei vollständiger Speisung im Normalbetrieb durch die zur Arbeitswelle benachbarte Feldoberwelle mit $v / p=-2$ dominiert. Die Verluste durch diese benachbarte Oberwelle in Höhe von $28.1 \mathrm{~kW}$ fallen im Vergleich zur Variante Z2 mit $q=2 / 5$ und $159 \mathrm{~kW}$ viel geringer aus, da der spektrale Abstand zwischen Arbeitswelle und benachbarter Harmonischen betragsmäBig zu Z2 um den Faktor 2.5 größer ist. Im Vergleich zu den anderen Zahnspulenvarianten treten sowohl im Normalbetrieb als auch bei Quadranten-Speisung im Redundanzbetrieb die geringsten Rotorwirbelstromverluste auf.

Die Wicklungsfaktoren der Arbeitswelle für die Wicklungsvarianten Z1 und Z2 liegen bei 0.866 bzw. 0.933. Daher wird mit der Zahnspulenwicklung Z3 ( $q=3 / 8,2 p=192)$ und einem Wicklungsfaktor von 0.945 auch eine Zahnspulenwicklung mit höherem Wicklungsfaktor betrachtet (s. Abb. 8 und Abb. 9). Allerdings verringert sich bei Z3 der spektrale Abstand zwischen Arbeitswelle und be-
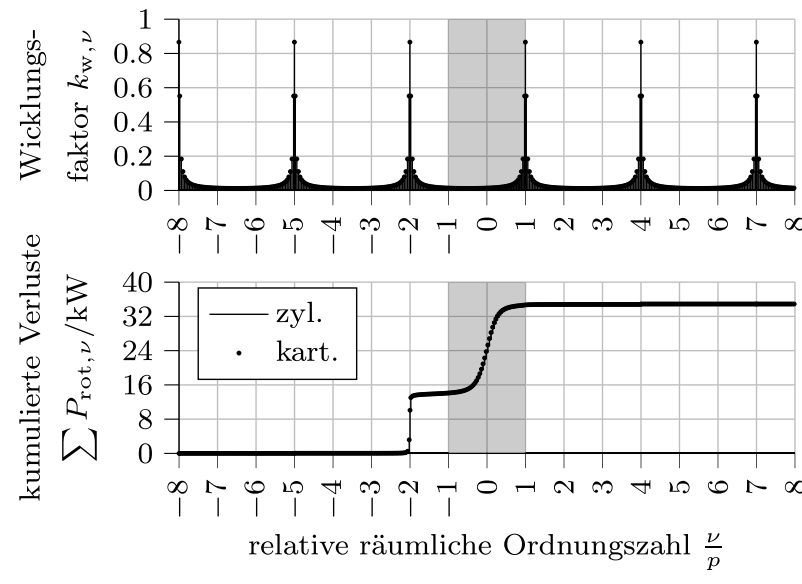

Abb. 7. Wicklungsvariante $Z 1(q=1 / 2,2 p=192)$, Redundanzbetrieb mit Quadranten-Speisung gemäß Abb. 1: Analytische Berechnung der Wirbelstromverluste im Rotorjoch

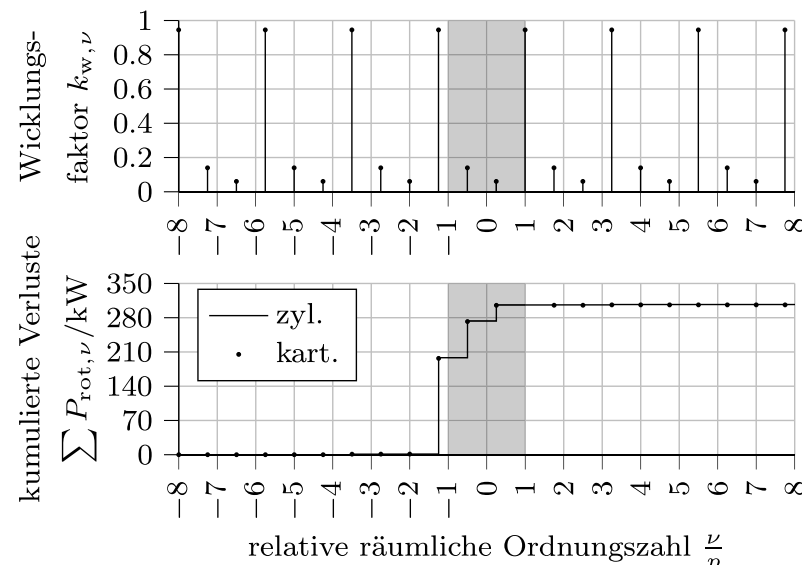

Abb. 8. Wicklungsvariante $Z 3(q=3 / 8,2 p=192)$, Normalbetrieb gemäß Tab. 2: Analytische Berechnung der Wirbelstromverluste im Rotorjoch

nachbarter Oberwelle auf $|v / p|-1=0.25$. Die zugehörigen Rotorwirbelstromverluste steigen auf $196 \mathrm{~kW}$. Überdies führen die beiden Unterwellen mit $v / p=0.25$ und $v / p=-0.5$ zu zusätzlichen Rotorwirbelstromverlusten von $33.3 \mathrm{~kW}$ bzw. $75.3 \mathrm{~kW}$. Die kumulierten Rotorwirbelstromverluste betragen im Normalbetrieb $307 \mathrm{~kW}$ und überschreiten die thermisch zulässigen Verluste um $23 \%$.

Eine andere Strategie zur Erzielung von möglichst hohen Wicklungsfaktoren nahe Eins bei möglichst wenigen Feldober- und Feldunterwellen ist die Erhöhung der Strangzahl $m$ der Statorwicklung. Gemäß den Annahmen stehen zwei dreiphasige Umrichter zur Verfügung, die bei einem Phasenversatz der Ausgangsdrehspannungssysteme von $30^{\circ}$ el. ein gemeinsames sechssträngiges Spannungssystem erzeugen. Damit ist auch die Implementierung der Wicklungsvariante $Z 4$ ( $m=6, q=1 / 5,2 p=180)$ möglich und wird in Abb. 10 und Abb. 11 dargestellt.

Vorteilhaft sind der hohe Wicklungsfaktor in Höhe von 0.966 sowie das Nichtauftreten von Unterwellen. Allerdings kann bei Ausfall eines Umrichters das sechsphasige System nicht aufrecht erhalten werden. Ein Redundanzbetrieb basiert deshalb auf der Speisung von drei verbleibenden Phasen eines Umrichters, verteilt am Gesamtumfang der Maschine. In diesem Betriebsmodus tritt eine Unterwelle 


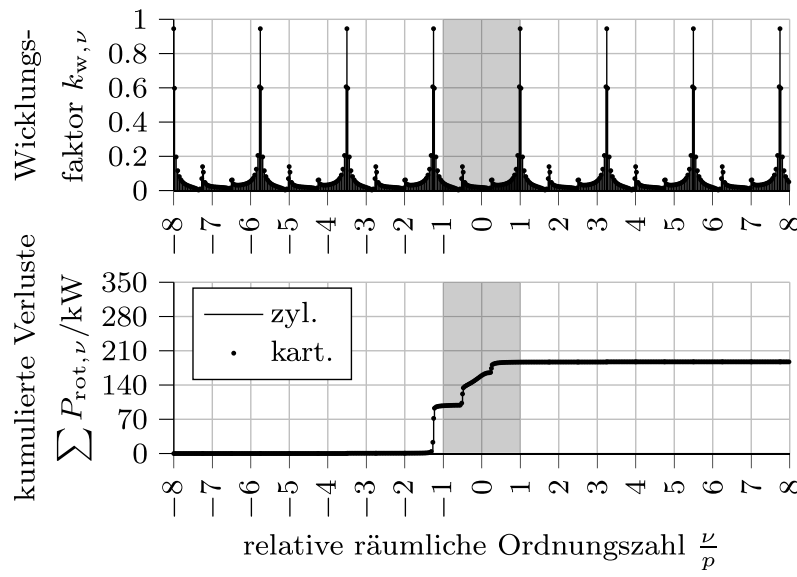

Abb. 9. Wicklungsvariante $Z 3(q=3 / 8,2 p=192)$, Redundanzbetrieb mit Quadranten-Speisung gemäß Abb. 1: Analytische Berechnung der Wirbelstromverluste im Rotorjoch

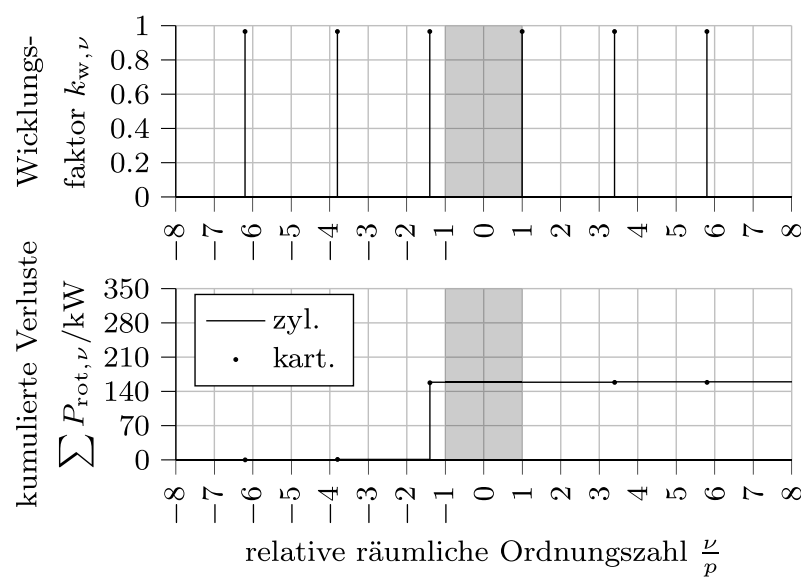

Abb. 10. Wicklungsvariante $Z 4(m=6, q=1 / 5,2 p=180)$, Normalbetrieb gemäß Tab. 2: Analytische Berechnung der Wirbelstromverluste im Rotorjoch

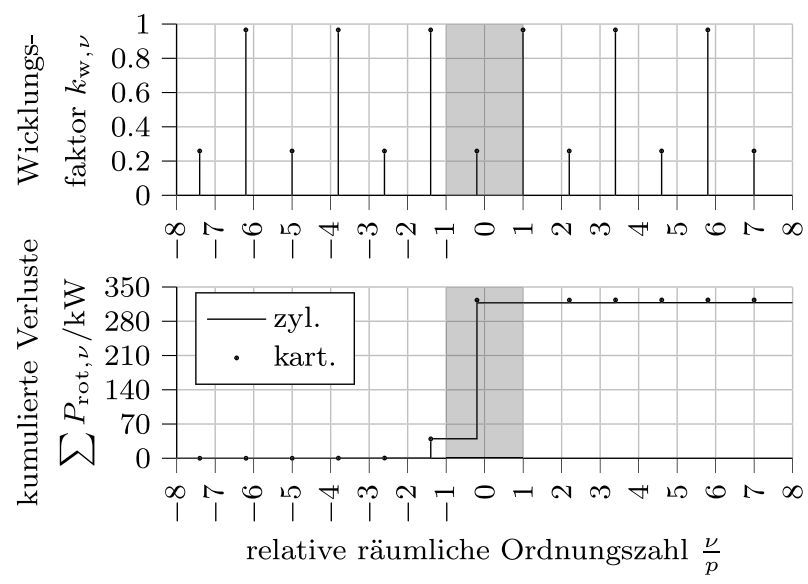

Abb. 11. Wicklungsvariante $\mathrm{Z4}(m=6, q=1 / 5,2 p=180)$, Redundanzbetrieb: Speisung von nur drei Strängen: Analytische Berechnung der Wirbelstromverluste im Rotorjoch
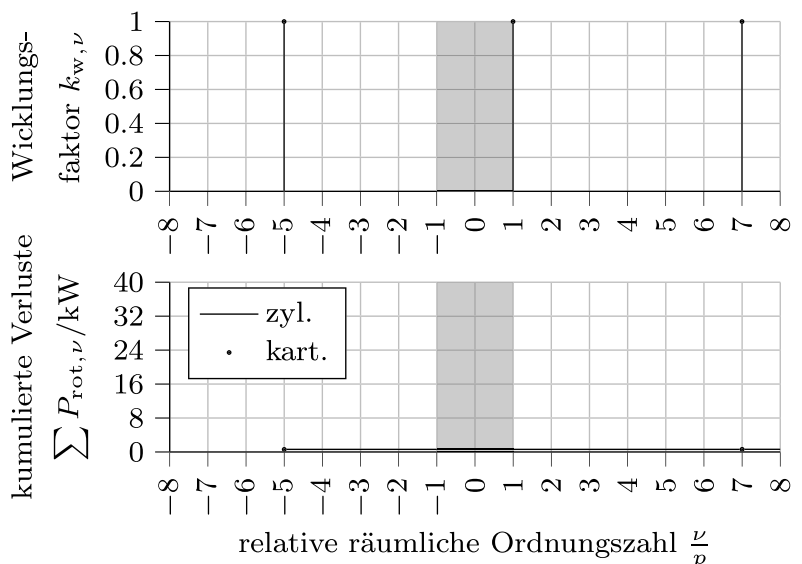

Abb. 12. Wicklungsvariante $\mathrm{V} 1(q=1,2 p=144)$, Normalbetrieb gemäß Tab. 2: Analytische Berechnung der Wirbelstromverluste im Rotorjoch

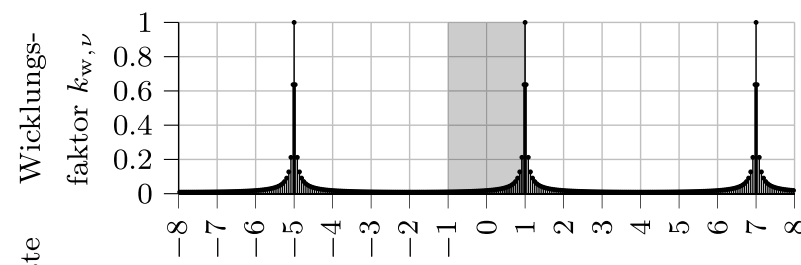

L

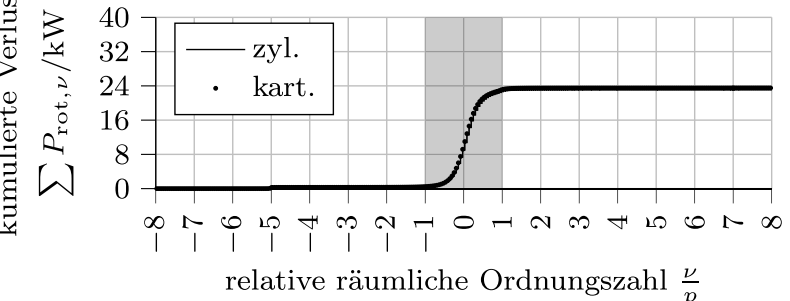

Abb. 13. V1 $(q=1,2 p=144)$, Redundanzbetrieb mit Quadranten-Speisung gemäß Abb. 1: Analytische Berechnung der Wirbelstromverluste im Rotorjoch

mit $v / p=-0.2$ auf, die allein schon zu unzulässig hohen Rotorjochwirbelstromverlusten von $277.7 \mathrm{~kW}$ führt.

In Abb. 12 und Abb. 13 werden die berechneten spektralen Rotorwirbelstromverlustverteilungen für die verteilte Wicklung V1 ( $q=1$, $2 p=144)$ gezeigt. Im Normalbetrieb treten nahezu keine Rotorwirbelstromverluste auf, da die Nutharmonischen als Feldoberwellen in einem Verhältnis $v / p=-5$ bzw. $v / p=7$ so kurzwellig sind, dass sie einerseits nur geringe fiktive Feldamplituden von $20 \%$ bzw. $14 \%$ der Arbeitswellenamplitude ermöglichen und andererseits kaum in der Lage sind, den im Vergleich zu den kurzen Wellenlängen relativ großen, magnetisch wirksamen Luftspalt zu überwinden. Bei der Quadranten-Speisung im Redundanzbetrieb führen die nun zusätzlich auftretenden Feldunterwellen zu unkritisch geringen Rotorwirbelstromverlusten in Höhe von $23.1 \mathrm{~kW}$.

\section{Optimierung des Blechschnitts}

\subsection{Optimierungsansatz}

Allgemein zeichnet sich eine optimale Maschine dadurch aus, dass sie bei minimalen Gesamtkosten die im Lastenheft geforderten Anforderungen unter Einhaltung gegebener Randbedingungen erfüllt. 
Tab. 3. Dimensionslose Eingangsparameter des Blechschnitts

\begin{tabular}{ll}
\hline Geometrieparameter & Formelzeichen \\
\hline Statorhöhe/(Statorhöhe + Rotorhöhe) & $0<f_{\mathrm{sr}}<1$ \\
Polbedeckungsfaktor der Magnete & $0<f_{\mathrm{p}} \leq 1$ \\
Magnethöhe/(Magnethöhe + Rotorjochhöhe) & $0<f_{\mathrm{mr}}<1$ \\
Nutbreite/(Nutbreite + Zahnbreite) & $0<f_{\mathrm{nz}}<1$ \\
Statorzahnlänge/(Statorzahnlänge + Statorjoch) & $0<f_{\mathrm{zj}}<1$ \\
\hline
\end{tabular}

Im vorliegenden Fall werden für die beiden Zahnspulenwicklungen Z1 und Z2 die Blechschnitte optimiert. Statt einer Gesamtkostenfunktion werden zwei Kriterien verwendet: Erstens der nachfolgend definierte genäherte Wirkungsgrad $\eta^{*}$ im Bemessungspunkt und zweitens die eingesetzte Magnetmasse $m_{\text {mag }}$, da diese hinsichtlich der Materialkosten ein wesentlicher Faktor ist. Ziel der Optimierung ist die Ermittlung der pareto-effizienten Maschinen im Hinblick auf diese Kriterien $[2,5]$. Die Blechschnittgeometrie jedes Individuums stellt die variable Eingangsgröße bzw. den Parametervektor da. Der Blechschnitt wird über fünf dimensionslose Längenverhältnisse in Tab. 3 beschrieben. Es handelt sich um eine Maschine mit Rechtecknuten und rechteckförmigen Oberflächenmagneten. Die axiale Blechpaketlänge wird unter Berücksichtigung der axialen Wickelkopfausladung aus der axial verfügbaren Gesamtlänge ermittelt und stellt damit ein wichtiges Optimierungspotential gegenüber verteilten Wicklungen mit ihren deutlich größeren Wickelköpfen dar. Zur Optimierung wird aufgrund der relativ hohen Anzahl von fünf Eingangsparametern ein multikriterieller genetischer Algorithmus verwendet [5], der in MATLAB implementiert ist [27].

Zur Ermittlung des Wirkungsgrads $\eta^{*}$ werden nichtlineare, FiniteElemente-Simulationen jedes Individuums mit der 2-D-Software FEMM [17] durchgeführt. Da es sich um einen nichtlinearen, magnetostatischen Löser handelt, ist die Drehung des Rotors zur Erfassung der Drehmomentwelligkeit separat implementiert. Zunächst werden für jedes Individuum die zur Erreichung des Bemessungspunktes $n_{N}, M_{N}$ (s. Tab. 4) notwendige Bestromung $\left|I_{s}\right|$ und der Bestromungswinkel $\beta=\angle\left\{\underline{U}_{\mathrm{p}} ; \underline{I}_{\mathrm{s}}\right\}$ als Winkel zwischen den Zeigern der Polradspannung je Strang $\underline{U}_{p}$ und dem Strangstrom $\underline{I}_{s}$ nach dem MTPA-Prinzip (Maximum Torque per Ampere) eingestellt. Zur Ermittlung des MTPA-optimalen $\left|\underline{I}_{s}\right|$ und $\beta$ kommt ein unterlagerter gradientenbasierter Optimierer zum Einsatz.

Der genäherte Wirkungsgrad $\eta^{*}$ bezieht sich auf den Bemessungspunkt mit MTPA-optimaler Bestromung $\left|\underline{I}_{s}\right|, \beta$ und berücksichtigt die beiden wesentlichen Verlustgruppen, nämlich die analytisch berechneten Stromwärmeverluste $P_{\mathrm{cu}, \mathrm{DC}}$ bei $\vartheta_{\mathrm{cu}}=100^{\circ} \mathrm{C}$ und die analytisch berechneten Wirbelstromverluste im Rotorjoch $P_{\text {rot }}$ gemäß Abschn. 2.1. Da es sich um einen langsam drehenden $\left(n_{N}=10.2 \mathrm{~min}^{-1}\right)$ Generator für eine getriebelose Windenergieanlage handelt, werden trotz der hohen Polzahl nur geringe elektrische Statorstromgrundfrequenzen (max. $17 \mathrm{~Hz}$ ) erreicht. Daher dominieren die Stromwärmeverluste die Verlustbilanz. Eine Maschine mit geringen Stromwärmeverlusten wird also im vorliegenden Fall tendenziell einen hohen Wirkungsgrad aufweisen, falls sie unter Berücksichtigung der stromabhängigen Eisensättigung in der Lage ist, das geforderte Drehmoment $M_{N}$ zu erreichen. Da während der Optimierung der Maschinengeometrie die Strangwindungszahl $N_{\mathrm{s}}$ und die Verschaltung der Wicklung noch nicht bekannt sind, wird zur Bestimmung der Stromwärmeverluste der warme Gleichstromwiderstand bei $\vartheta_{\mathrm{cu}}=100{ }^{\circ} \mathrm{C}$ verwendet. Im Unterschied zur Vorausberechnung mit geschätzter Geometrie und geschätztem Statorstrombelag in Abschn. 2.2, wird hier die tatsächliche Geometrie und die
Tab. 4. Randbedingungen für die Optimierung

\begin{tabular}{|c|c|c|}
\hline & Symbol & Wert \\
\hline Bemessungsdrehmoment & $M_{\mathrm{N}}$ & $8.3 \mathrm{MNm}$ \\
\hline Bemessungsdrehzahl & $n_{N}$ & $10.2 \mathrm{~min}^{-1}$ \\
\hline Bemessungsspannung (verkettet) & $U_{N}$ & $820 \mathrm{~V}$ \\
\hline Verschaltung & & Stern \\
\hline Rotoraußendurchmesser & $d_{r, 0}$ & $6500 \mathrm{~mm}$ \\
\hline Statorinnendurchmesser & $d_{\mathrm{s}, \mathrm{i}}$ & $6084.4 \mathrm{~mm}$ \\
\hline Mechanischer Luftspalt & $\delta$ & $8 \mathrm{~mm}$ \\
\hline $\begin{array}{l}\text { Axiale Gesamtlänge ohne Wickelkopf } \\
\text { auf der Verschaltungsseite }\end{array}$ & $l_{\mathrm{ax}, \mathrm{ss}}$ & $1872 \mathrm{~mm}$ \\
\hline Anzahl radialer Kühlschlitze & $n_{\mathrm{k}}$ & 22 \\
\hline Breite der Kühlschlitze & $l_{\mathrm{k}}$ & $5 \mathrm{~mm}$ \\
\hline Rotormaterial & \multirow{2}{*}{\multicolumn{2}{|c|}{$\begin{array}{c}\text { Unlegierter Stahl (S10C) } \\
\text { M470P-65A }\end{array}$}} \\
\hline Statorblech & & \\
\hline Magnetmaterial & \multicolumn{2}{|c|}{$\mathrm{NdFeB}(\mathrm{N} 46 \mathrm{H})$} \\
\hline Geschätzte Magnettemperatur & $\vartheta_{\text {mag }}$ & $50^{\circ} \mathrm{C}$ \\
\hline Remanenzflussdichte $\vartheta_{\text {mag }}$ & $B_{\mathrm{r}}$ & $1.31 \mathrm{~T}$ \\
\hline Relative Permeabilität & $\mu_{r, \text { mag }}$ & 1.04 \\
\hline Dichte & $\rho_{\text {mag }}$ & $7550 \mathrm{~kg} / \mathrm{m}^{3}$ \\
\hline Geschätzte Leitertemperatur & $\vartheta_{\mathrm{cu}}$ & $100^{\circ} \mathrm{C}$ \\
\hline Geschätzter Kupferfüllfaktor & $k_{\mathrm{cu}}$ & $88.2 \%$ \\
\hline
\end{tabular}

aus der MTPA-Optimierung gewonnene Statorbestromung bei der Rotorwirbelstromverlustberechnung verwendet.

Die Randbedingungen des Optimierungsproblems sind in Tab. 4 aufgeführt und umfassen folgende Restriktionen:

1. Luftspalt:

Aufgrund der großen Maschinenabmessungen und der geringen Rotorjochhöhen ergeben sich bei entsprechender Radialkraftanregung relativ große radiale Verformungen des Rotors, so dass ein ausreichend großer mechanischer Luftspalt von $\delta=$ $8 \mathrm{~mm}$ fest vorgegeben ist.

2. Bauraum:

Der Bauraum der Maschine wird als ringförmiges Volumen vorgegeben, das durch den Statorinnendurchmesser $d_{s, i}$ und den Rotoraußendurchmesser $d_{r, o}$ sowie die axiale Länge $l_{a x, s s}$ charakterisiert ist. Die vorgegebene axiale Länge $l_{\mathrm{ax}, \text { ss }}$ umfasst dabei die Blechpaketlänge mit radialen Kühlschlitzen und die axiale Wickelkopfausladung auf der Antriebsseite. Auf der Nichtantriebsseite befindet sich die Verschaltung der Statorwicklung, die mehr axialen Bauraum einnimmt als der Wickelkopf selbst und damit nicht zur freien Ausgestaltung der Maschine zur Verfügung steht.

3. Materialien:

Die Materialien für Magnete, Elektroblech, Rotorjoch und das Leitermaterial der Wicklung sind mit ihren elektromagnetischen Eigenschaften fest vorgegeben.

4. Statorwicklung:

Da während der Optimierung des Blechschnitts keine Wicklungsauslegung erfolgt, werden ein konstanter Kupferfüllfaktor $k_{\mathrm{cu}}$ und eine konstante mittlere Wicklungstemperatur $\vartheta_{\mathrm{cu}}$ vorgegeben.

\subsection{Optimierungsergebnisse: Pareto-Fronten}

In Abb. 14 und Abb. 15 sind die simulierten Individuen und die daraus resultierenden Pareto-Fronten für die Zahnspulenwicklungen Z1 


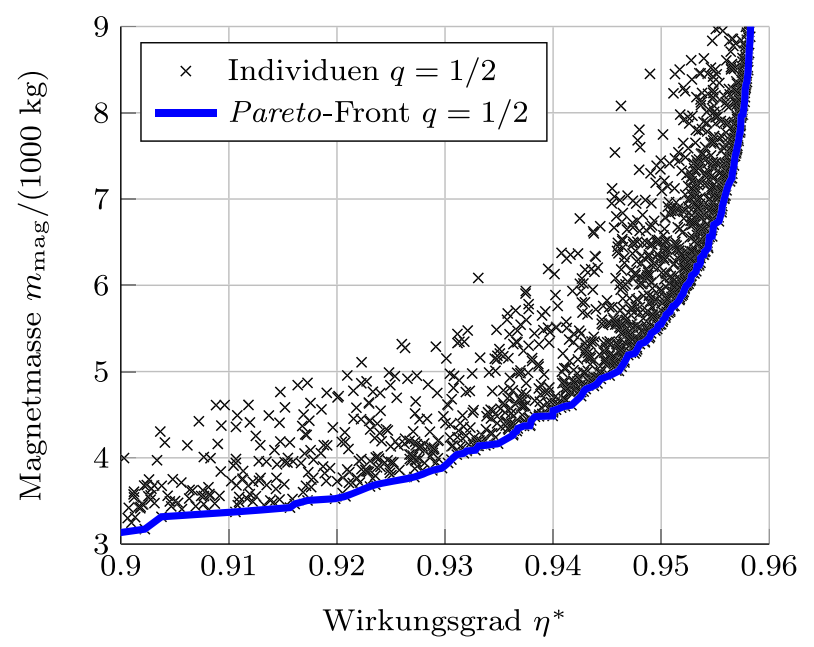

Abb. 14. Wicklungsvariante $Z 1$ ( $q=1 / 2$ und $2 p=192)$ : 1789 berechnete Individuen und Pareto-Front (Farbabbildung online)

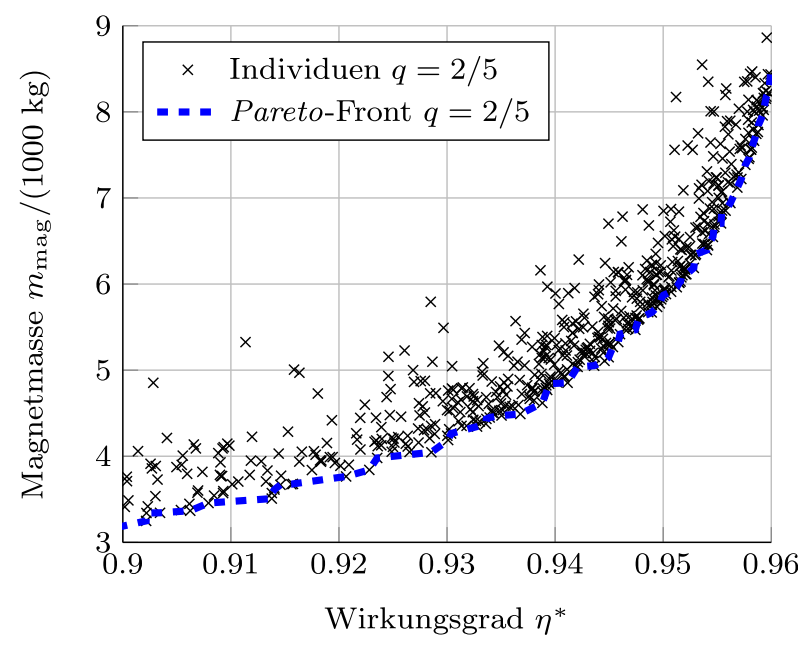

Abb. 15. Wicklungsvariante $Z 2(q=2 / 5$ und $2 p=180)$ : 940 berechnete Individuen und Pareto-Front (Farbabbildung online)

und Z2 dargestellt. Wie erwartet kann in beiden Fällen der Wirkungsgrad $\eta^{*}$ durch mehr Magnetmasse gesteigert werden. Allerdings nimmt der Grenznutzen des Magnetmaterialeinsatzes dabei kontinuierlich ab. Der nahezu glatte Verlauf der Pareto-Front weist auf die gute Konvergenz der numerischen Suche hin.

In Abb. 16 sind die Verläufe der Pareto-Fronten aus Abb. 14 und Abb. 15 gemeinsam dargestellt. In einem Bereich der Magnetmasse $m_{\text {mag }}$ von $3000 \mathrm{~kg}$ bis ca. $6700 \mathrm{~kg}$ hat die Wicklung Z1 mit $q=1 / 2$ einen höheren Wirkungsgrad $\eta^{*}$ als Wicklung $Z 2$ mit $q=2 / 5$. Bei mehr als $6700 \mathrm{~kg}$ Magnetmasse weist die Wicklung Z2 mit $q=2 / 5$ einen höheren Wirkungsgrad $\eta^{*}$ auf. Aus den pareto-effizienten Maschinen werden zwei Maschinen Z1 und Z2 mit einer Magnetmasse von $m_{\text {mag }}=4344 \mathrm{~kg}$ ausgewählt (Abb. 16), deren Berechnungsdaten in Tab. 5 dargestellt sind. Die Blechschnitte der beiden Maschinen weisen ähnliche Abmessungen auf. Allerdings benötigt $Z 1(q=1 / 2)$ einen deutlich höheren Strombelag und eine deutlich höhere Stromdichte im Vergleich zu Z2 ( $q=2 / 5)$ zum Erreichen des Bemessungsdrehmoments aufgrund des geringeren Wicklungsfaktors (vgl. Tab. 1). Die Verlustbilanz in Tab. 5 zeigt, dass die daraus resultierenden höheren Stromwärmeverluste von Z1 ( $q=1 / 2)$ im Ver-

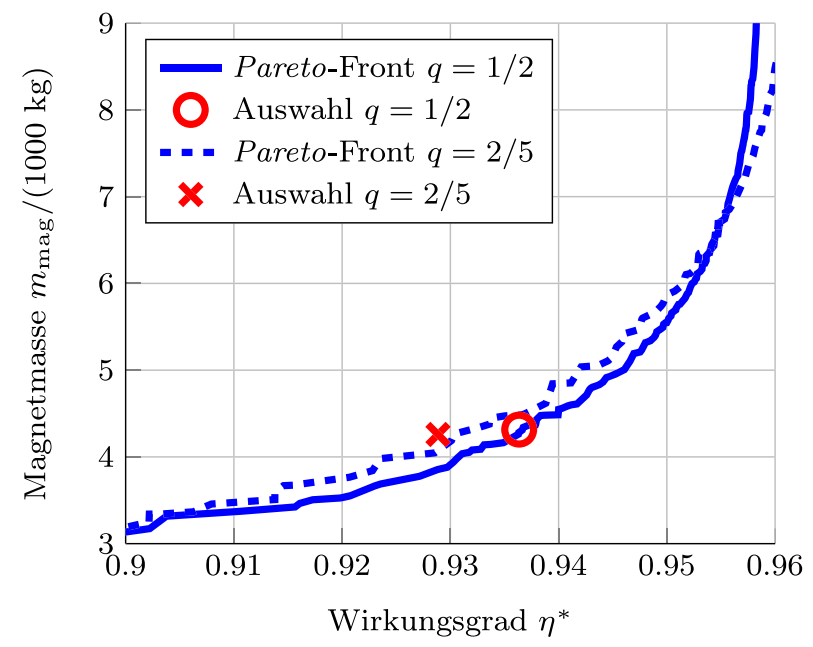

Abb. 16. Vergleich der Pareto-Fronten und der beiden ausgewählten Individuen mit gleicher Magnetmasse $m_{\mathrm{mag}}=\mathbf{4 3 4 4} \mathbf{~ k g}$ (Farbabbildung online)

Tab. 5. Ergebnis der Optimierung für zwei ausgewählte Individuen mit $m_{\text {mag }}=4344 \mathrm{~kg}$ (Abb. 16)

\begin{tabular}{lll}
\hline & Z1 & Z2 \\
\hline Lochzahl $q$ & $1 / 2$ & $2 / 5$ \\
Polzahl $2 p$ & 192 & 180 \\
Statorbemessungs-frequenz $f_{\mathrm{N}}$ & $16.3 \mathrm{~Hz}$ & $15.3 \mathrm{~Hz}$ \\
$f_{\mathrm{sr}}$ & 0.798 & 0.805 \\
$f_{\mathrm{p}}$ & 0.849 & 0.848 \\
$f_{\mathrm{mr}}$ & 0.462 & 0.474 \\
$f_{\mathrm{nz}}$ & 0.477 & 0.463 \\
$f_{\mathrm{zj}}$ & 0.846 & 0.812 \\
Statoraußendurchmesser $d_{\mathrm{s}, \mathrm{O}}$ & $6403 \mathrm{~mm}$ & $6406 \mathrm{~mm}$ \\
Rotorjochinnendurchmesser $d_{\mathrm{r}, \mathrm{i}}$ & $6456.2 \mathrm{~mm}$ & $6459 \mathrm{~mm}$ \\
Magnethöhe $h_{\mathrm{m}}$ & $18.6 \mathrm{~mm}$ & $18.5 \mathrm{~mm}$ \\
Blechpaketlänge $L$ mit radialen & $1804 \mathrm{~mm}$ & $1792 \mathrm{~mm}$ \\
Kühlkanälen & & \\
MTPA: Effektiver Strombelag & $1623 \mathrm{~A} / \mathrm{cm}^{2}$ & $1361 \mathrm{~A} / \mathrm{cm}^{2}$ \\
MTPA: Stromdichte (Effektivwert) & $3.45 \mathrm{~A} / \mathrm{mm}^{2}$ & $2.90 \mathrm{~A} / \mathrm{mm}^{2}$ \\
MTPA: Stromvorsteuerwinkel $\beta$ & $162^{\circ}$ & $167^{\circ}$ \\
MTPA: $P_{\text {cu,DC }}$ & $521 \mathrm{~kW}$ & $381 \mathrm{~kW}$ \\
MTPA: Prot $_{\text {MTPA: } \eta^{*}}$ & $43.7 \mathrm{~kW}$ & $249 \mathrm{~kW}$ \\
\hline
\end{tabular}

gleich zu Z2 ( $q=2 / 5)$ durch die deutlich geringeren Rotorjochwirbelstromverluste kompensiert werden. Demnach liegt der analytisch genäherte Wirkungsgrad $\eta^{*}$ von der gewählten Maschine Z1 um 0.7 Prozentpunkte höher als bei der gewählten Maschine Z2.

\section{Wicklungsauslegung und transiente FEM-Simulation}

Für die beiden in Tab. 5 aufgeführten ausgewählten Individuen erfolgt nun die detaillierte Wicklungsauslegung. Mit diesen Daten wird mit transienten FEM-Simulationen (Berechnungsprogramm $J M A G)$ der Wirkungsgrad im Bemessungspunkt ermittelt. Dabei werden die Wirbelstromverluste im Rotorjoch $P_{\text {rot }}$ und in den Magneten $P_{\text {mag }}$ numerisch und nicht wie bisher analytisch berechnet. Auch die Ummagnetisierungsverluste im Statorblechpaket $P_{\mathrm{Fe}, \mathrm{s}}$ und 
Tab. 6. Wicklungsauslegung und Ergebnisse der FEM-Nachrechnung für den Bemessungspunkt (MTPA)

\begin{tabular}{|c|c|c|}
\hline & $\mathrm{Z1}$ & $\mathrm{Z2}$ \\
\hline Spulenwindungszahl $N_{\mathrm{C}}$ & 31 & 25 \\
\hline $\begin{array}{l}\text { Parallele Teilleiter je Windung } \\
\text { (nebeneinander) } a_{i}\end{array}$ & 2 & 2 \\
\hline Parallele Zweige $a_{a}$ je Strang & 48 & 36 \\
\hline Strangwindungszahl $N_{s}$ & 62 & 50 \\
\hline Teilleiter blank: Höhe & $3.84 \mathrm{~mm}$ & $4.63 \mathrm{~mm}$ \\
\hline Teilleiter blank: Breite & $7.03 \mathrm{~mm}$ & $9.49 \mathrm{~mm}$ \\
\hline Teilleiterfläche (blank) & $26.5 \mathrm{~mm}^{2}$ & $43.8 \mathrm{~mm}^{2}$ \\
\hline $\begin{array}{l}\text { Anzahl axialer } \\
\text { Magnetsegmentierungen }\end{array}$ & 40 & 40 \\
\hline $\begin{array}{l}\text { Anzahl radialer } \\
\text { Magnetsegmentierung }\end{array}$ & 3 & 3 \\
\hline $\begin{array}{l}\text { Strangspannung } U_{s, 1} \\
\text { (Grundschwingungseffektivwert) }\end{array}$ & $455.7 \mathrm{~V}$ & $448.9 \mathrm{~V}$ \\
\hline $\begin{array}{l}\text { Strangstrom } I_{\mathrm{s}, 1} \\
\text { (Grundschwingungseffektivwert) }\end{array}$ & $8778 \mathrm{~A}$ & $9130 \mathrm{~A}$ \\
\hline $\begin{array}{l}\text { Mechanisch zugeführte Leistung } \\
P_{\text {mech }}\end{array}$ & $8.93 \mathrm{MW}$ & $9.03 \mathrm{MW}$ \\
\hline Mittleres Drehmoment $\bar{m}$ & $-8.36 \mathrm{MNm}$ & $-8.45 \mathrm{MNm}$ \\
\hline Drehmomentwelligkeit $\Delta m /(2 \bar{m})$ & $7.0 \%$ & $0.3 \%$ \\
\hline $\begin{array}{l}\text { AC-Stromwärmeverluste } \\
\text { Statorwicklung } P_{\mathrm{Cu}, \mathrm{DC}}+P_{\mathrm{ad}, \mathrm{s}}\end{array}$ & $548 \mathrm{~kW}$ & $408 \mathrm{~kW}$ \\
\hline $\begin{array}{l}\text { Wirbelstromverluste im Rotorjoch } \\
P_{\text {rot }}\end{array}$ & $18.2 \mathrm{~kW}$ & 183 kW \\
\hline $\begin{array}{l}\text { Wirbelstromverluste in den } \\
\text { Magneten } P_{\text {mag }}\end{array}$ & $9.0 \mathrm{~kW}$ & $15.1 \mathrm{~kW}$ \\
\hline $\begin{array}{l}\text { Stator-Ummagnetisierungs- } \\
\text { verluste } P_{\mathrm{Fe}, \mathrm{s}}\end{array}$ & $53.2 \mathrm{~kW}$ & $48.5 \mathrm{~kW}$ \\
\hline $\begin{array}{l}\text { Gesamtverluste } P_{\mathrm{d}}=P_{\mathrm{cu}, \mathrm{DC}}+ \\
P_{\mathrm{ad}, \mathrm{s}}+P_{\mathrm{rot}}+P_{\mathrm{mag}}+P_{\mathrm{Fe}, \mathrm{s}}\end{array}$ & $628 \mathrm{~kW}$ & $655 \mathrm{~kW}$ \\
\hline El. Abgabeleistung & $8.30 \mathrm{MW}$ & $8.37 \mathrm{MW}$ \\
\hline Wirkungsgrad $\eta=1-P_{\mathrm{d}} / P_{\text {mech }}$ & $93.0 \%$ & $92.8 \%$ \\
\hline $\begin{array}{l}\text { Grundschwingungsleistungs- } \\
\text { faktor } \cos \left(\varphi_{1}\right)\end{array}$ & 0.69 (ind.) & 0.68 (ind.) \\
\hline
\end{tabular}

die Stromverdrängungsverluste $P_{\mathrm{ad}, \mathrm{s}}$ in der Statorwicklung werden durch diese transiente Simulation bestimmt. Interessant ist der Vergleich der hier ermittelten Verluste mit den zuvor geschätzten Verlusten $P_{\mathrm{Cu}, \mathrm{DC}}$ und $P_{\text {rot }}$, da die entsprechenden Simulationsmodelle und die eingesetzte Software unabhängig voneinander arbeiten. Darüber hinaus wird die Entmagnetisierfestigkeit im Kurzschlussfall numerisch mit JMAG überprüft.

Die Wicklungsauslegung erfolgt so, dass die in Tab. 2 vorgegebene Bemessungsspannung $U_{N}=820 \mathrm{~V}$ eingehalten wird und die Stromverdrängung in den Nutenleitern zu einer moderaten Widerstandserhöhung von weniger als 10 \% führt. Das Ergebnis der ungeschrägten Wicklungsauslegung für Z1 und Z2 ist in Tab. 6 aufgeführt. Zur hinreichenden Begrenzung der Wirbelstromverluste in den elektrisch leitfähigen Magneten wird für beide Varianten Z1 und Z2 eine axiale Segmentierung von 40 und drei Unterteilungen in Umfangsrichtung je Pol gewählt. Die resultierenden JMAG-Modelle von je einem ganzen bzw. einem halben Urwicklungsschema sind in Abb. 17 dargestellt.

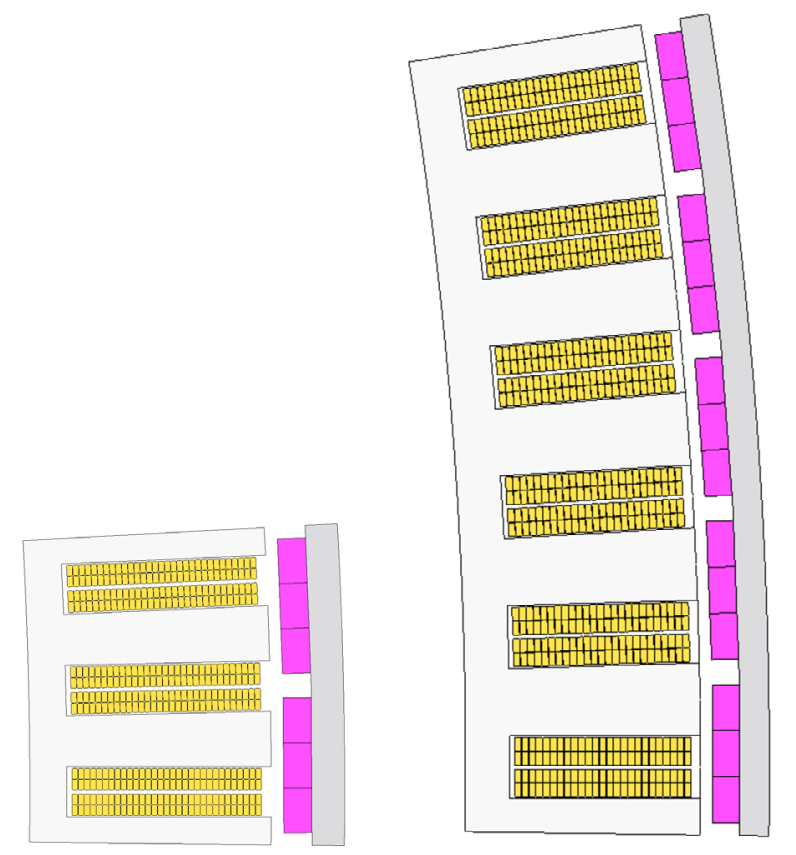

(a) Maschine Z1 $q=1 / 2$ : (b) Maschine Z2 $q=2 / 5$ : $J M A G$-Modell (ein Urwi- JMAG-Modell (halbes Urwickeckelungsschema) lungsschema)

Abb. 17. JMAG-Modelle der ausgewählten Maschinen (Farbabbildung online)

Die Auswertung der 2D-Lastsimulation im Bemessungspunkt (MTPA) bei Grundschwingungsstromspeisung in Tab. 6 ergibt deutlich geringere Wirbelstromverluste als die analytische Berechnung in Tab. 5. Der Grund hierfür liegt in der Annahme magnetisch linearer Medien im analytischen Modell. Der numerisch ermittelte Gesamtwirkungsgrad $\eta$ liegt für beide Maschinen aufgrund der zusätzlich betrachteten Verlustgruppen $P_{\mathrm{ad}, s}, P_{\mathrm{Fe}, \mathrm{s}}$ und $P_{\mathrm{mag}}$ um 0.6 Prozentpunkte bei Maschine Z1 und um 0.1 Prozentpunkt bei Maschine Z2 niedriger als der geschätzte Wirkungsgrad $\eta^{*}$. Aus den transienten Simulationen wird auch der Spitze-Spitze-Wert des DrehmomentZeitverlaufs $\Delta m$ ermittelt. Die Maschine Z1 weist mit $7 \%$ eine deutlich höhere Drehmomentwelligkeit auf als die Maschine Z2 mit $0.3 \%$. Grund hierfür ist das hohe Rastmoment bei Maschine Z1 $(q=1 / 2)$, da das kleinste gemeinsame Vielfache der Polzahl und Nutzahl relativ gering ist im Verhältnis zur Polpaarzahl [13]. Daraus resultiert für Z1 $(q=1 / 2)$ eine Drehmomentwelligkeit mit der sechsfachen Statorstromgrundfrequenz $97.8 \mathrm{~Hz}$. Bei Maschine $\mathrm{Z} 2(q=2 / 5)$ tritt erst das 12-fache der Statorstromgrundfrequenz 183.6 Hz als Drehmomentwelligkeit durch Nutrasten auf, wodurch die Amplitude der Drehmomentwelligkeit um den Faktor 23 geringer ist.

Der Nachweis der Entmagnetisierfestigkeit der Magnete bei Stoßkurzschluss aus vorhergehendem generatorischen Bemessungsbetrieb bei betriebswarmen Magneten $\left(\vartheta_{\text {mag }}=50^{\circ} \mathrm{C}\right)$ wurde mit Hilfe einer weiteren Finite-Elemente-Simulation für beide Maschinen erbracht. Aufgrund des im Vergleich zur Polteilung $\tau_{\mathrm{p}}$ großen magnetisch wirksamen Luftspalts $\left(b / \tau_{\mathrm{p}} \approx 0.28\right)$ ist der Stoßkurzschluss unkritisch und wird hier nicht detailliert vorgestellt.

\section{Geräuschanregung}

Aus Platzgründen kann nur kurz auf die möglichen Radialschwingungsanregungen des Stator- und Rotorjochs eingegangen wer- 
den. Für eine genauere Analyse müssen die mechanischen Eigenfrequenzen und zugehörigen modalen Schwingungsformen der Statorund Rotorjoch-Biegeschwingungen numerisch mit Berücksichtigung der dreidimensionalen Geometrie, der Materialdämpfung, der Einspannkräfte und des Einflusses der Wicklung ermittelt werden [9, 29]. Hier werden diese Eigenfrequenzen mit dem Schwingungsmodell des homogenen Rings nur grob abgeschätzt [14], um einen ersten Eindruck der möglichen Schwingungsanregung zu gewinnen. Weiter wird der Einfluss der Stromoberschwingungen auf die magnetische Geräuschbildung hier nicht betrachtet.

Aus der Finite-Elemente-Simulation mit Stromgrundschwingungsspeisung wird die Radialkomponente des Maxwell'schen Spannungstensors in der Luftspaltmitte berechnet. Näherungsweise kann diese Größe als Zugkraftverteilung auf den luftspaltseitigen Oberflächen von Rotor und Stator bei zentrischer Rotorlage aufgefasst werden. Allgemein ist diese Näherung nicht zulässig, da der Maxwell'sche Spannungstensor keine Kraftlokalisierung zulässt, sondern nur zur Bestimmung der Summenkraft auf einen Körper herangezogen werden kann [12]. Mit der vereinfachenden Annahme von unendlich permeablen Eisenteilen tritt jedoch ausschließlich eine lokale Kraftwirkung an den Eisenoberflächen auf.

\subsection{Radialkraftanregung für Maschine Z1 $(q=1 / 2)$}

In Abb. 18 ist die zeitliche und räumliche Verteilung der numerisch ermittelten Radialkomponente des Maxwell'schen Zugs in der Luftspaltmitte für Maschine Z1 im Bemessungspunkt über eine zeitliche Periode $T_{\mathrm{s}}$ und räumliche Periode $2 \tau_{\mathrm{p}}$ in statorfesten Koordinaten dargestellt. Deutlich zu erkennen ist die Kraftkonzentration an den drei Zähnen durch die vertikalen dunklen Bereiche hoher Zugkraft. Diese Daten wurden spektral mit Hilfe der zweidimensionalen diskreten Fourier-Reihenentwicklung ausgewertet. Die zeitliche Ordnungszahl $k_{\mathrm{s}}$ bezieht sich auf die elektrische Statorgrundfrequenz und die räumliche Ordnungszahl $v$ auf den Gesamtumfang der Maschine. Das resultierende statorfeste Kraftwellenspektrum ist in Abb. 19 dargestellt. Für das rotorseitige System ergibt sich eine Verschiebung der zeitlichen Kraftwellenordnungen $k_{r}$ auf der Rotorseite aufgrund der Drehbewegung nach (24) analog zu (7); die Amplituden bleiben unverändert.

$$
k_{\mathrm{r}}=k_{\mathrm{s}}-\frac{v}{p}
$$

Bei zentrischer Läuferlage ergibt sich aus der Simulation nur eine relevante Radialkraftanregung. Diese hat die räumliche Ordnung $v=0$ und die zeitliche Ordnung $k_{\mathrm{s}}=6$. Weitere Anregungen treten bei ganzzahligen Vielfachen der Polpaarzahl $p$ auf. Da $p=96$ relativ groß ist, wird davon ausgegangen, dass das Blechpaket keine nennenswert großen Verformungsamplituden mit diesen hohen Ordnungszahlen ausbildet [29], da sich der Frequenzabstand zwischen Anregung und Biegeeigenfrequenz mit steigender räumlicher Ordnung vergrößert: Die Biegeeigenfrequenzen des Jochrings steigen näherungsweise quadratisch mit der räumlichen Ordnung an [14], während die Anregungsfrequenzen nur linear mit der räumlichen Ordnung zunehmen (s. Abb. 19).

Die Amplituden der Kraftwelle mit $v=0$ und $k_{s}=6$ betragen bei Bemessungslast $5.6 \mathrm{kPa}$ und im generatorischen Leerlauf $4.3 \mathrm{kPa}$. Die hohe Kraftamplitude im Leerlauf zeigt, dass die Modulation des PM-Läuferfelds durch die offenen Statornuten einen großen Beitrag zur Radialkraftanregung leistet. Da für die räumliche Ordnungszahl $v=0$ keine Änderung der zeitlichen Ordnungszahl beim Übergang in das Rotorkoordinatenystem erfolgt, gelten diese Kraftanregungsamplituden und die zeitliche Ordnung $k_{\mathrm{s}}=k_{\mathrm{r}}=6$ auch für das Rotorjoch.

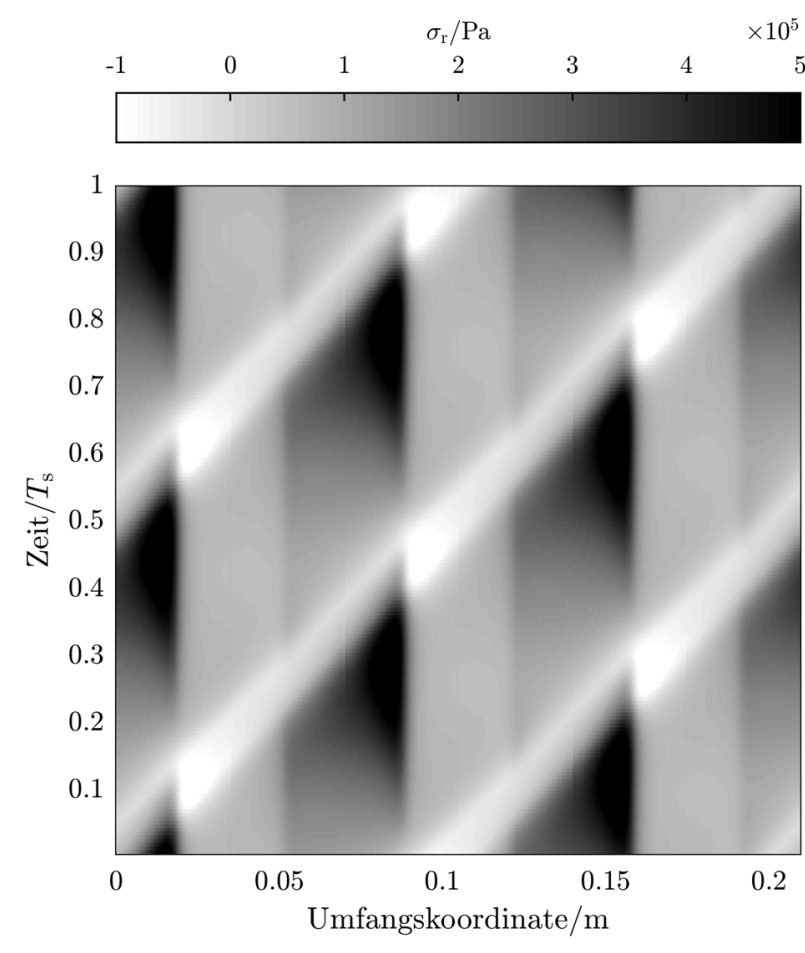

Abb. 18. Maschine Z1, Bemessungslast: Verlauf der berechneten Radialkomponente des Maxwell'schen Zugs längs der Luftspaltmitte (JMAG-Simulation)

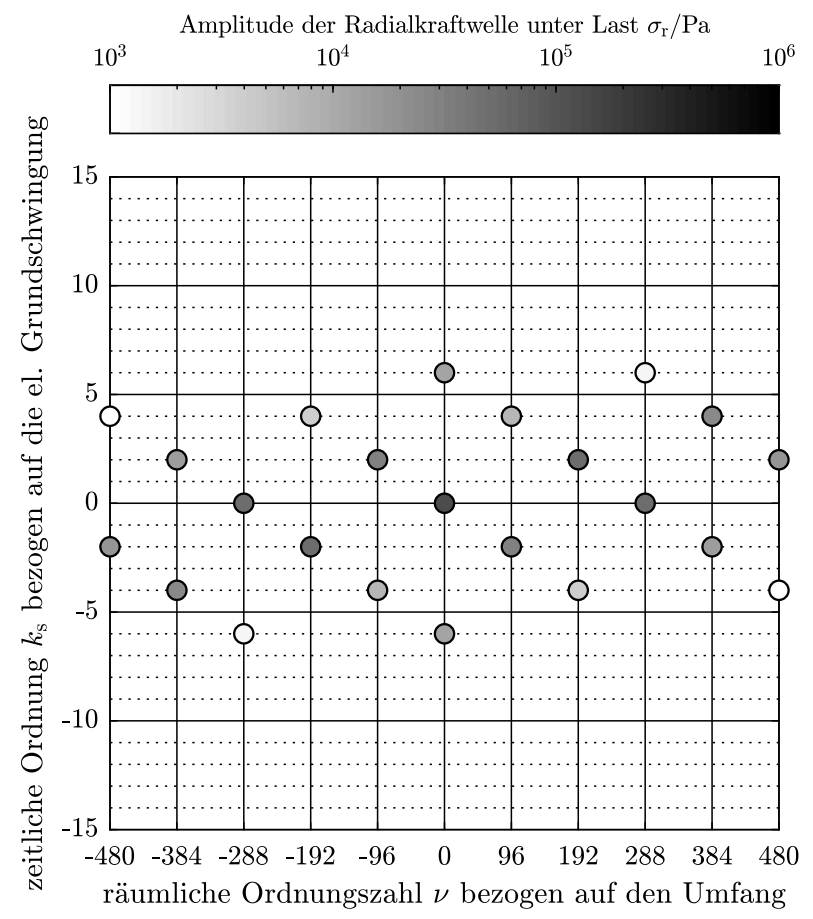

Abb. 19. Maschine Z1, Bemessungslast: Spektrum der berechneten Radialkomponente des Maxwell'schen Zugs längs der Luftspaltmitte

Zur Beurteilung möglicher Schwingungsanregungen der Eigenformen von Stator- und Rotorjoch sind die Anregungsfrequenzen mit den Biegeeigenfrequenzen von Rotor- und Statorjoch zu verglei- 


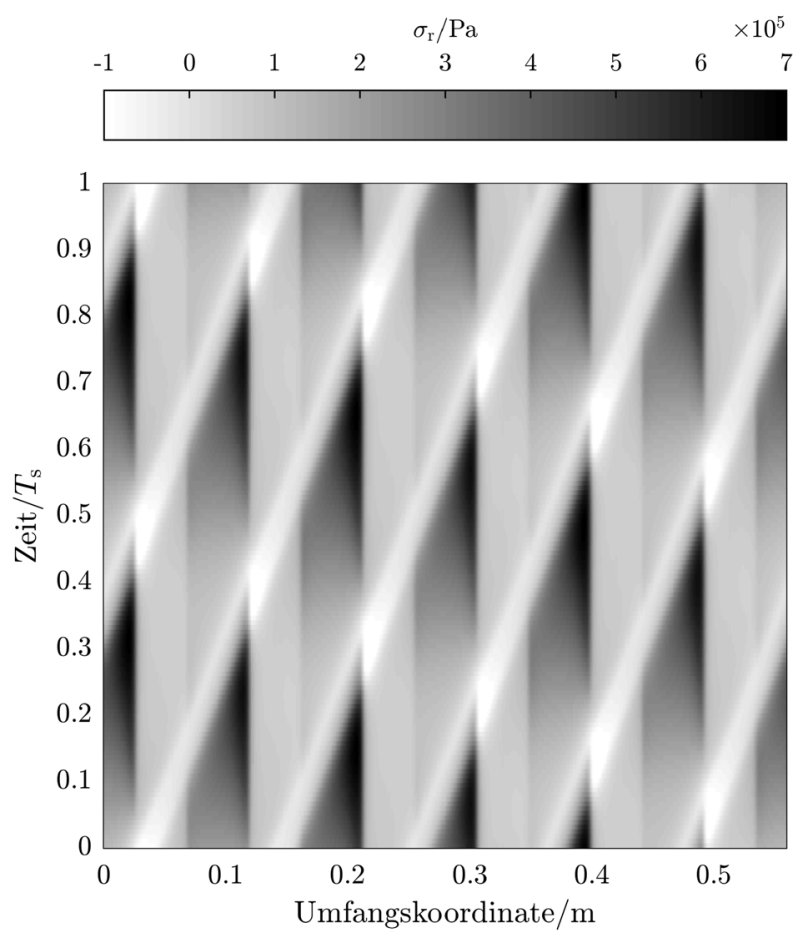

Abb. 20. Maschine Z2, Bemessungslast: Verlauf der errechneten Radialkomponente des Maxwell'schen Zugs längs der Luftspaltmitte (JMAG). Dargestellt ist ein halbes Urschema der Maschine bestehend aus 6 Zähnen bzw. 5 Polen

chen. Der Drehzahlbereich $0 \ldots n_{N}$ führt drehzahlabhängig auf Anregungen der nullten Biegeschwingungsmode im Frequenzbereich von $f_{\min }=0 \mathrm{~Hz}$ bis $f_{\max }=k_{\mathrm{s} / \mathrm{r}} \cdot f_{\mathrm{N}}=97.92 \mathrm{~Hz}$. Nach [14] werden die Biegeeigenfrequenzen der nullten Biegeschwingungsmode für das Statorjoch zu $f_{\mathrm{s}, \text { eig, } 0}=106.8 \mathrm{~Hz}$ und für das ringförmige Rotorjoch zu $f_{\mathrm{r}, \text { eig, } 0}=194.9 \mathrm{~Hz}$ berechnet. Damit ergibt sich zwischen Anregungs- und Eigenfrequenz des Statorjochs ein Frequenzabstand von nur 0.04 Dekaden. Nach dieser Berechnung ist davon auszugehen, dass bei Bemessungsdrehzahl die nullte Biegeeigenschwingungsform des Statorjochs angeregt wird und zu hohen Radialschwingungsamplituden führt. Allerdings ist zu beachten, dass der Einfluss des Innengehäuses des Statorblechpakets sowohl hinsichtlich der Versteifung als auch Zusatzmasse bei der Abschätzung der Resonanzfrequenzen nach [14] nicht berücksichtigt wurde, sondern nur der Einfluss der Zahn-, Wicklungs- bzw. Magnetmassen als Zusatzmasse für das Stator- bzw. Rotorjoch.

\subsection{Radialkraftanregung für Maschine $Z 2(q=2 / 5)$}

Zunächst wird aus dem numerisch ermittelten räumlichen und zeitlichen Verlauf der Radialkomponente des Maxwell'schen Spannungstensors in Abb. 20 das Spektrum der Radialkraftwellen in Abb. 21 ermittelt. Besonders kritisch ist wiederum die Radialkraftwelle mit der geringsten räumlichen Ordnungszahl $|v|=36$ mit einer zeitlichen Ordnungszahl im Stator $\left|k_{\mathrm{s}}\right|=2$ und im Rotor $\left|k_{\mathrm{r}}\right|=2.4$

Die Amplitude der Radialkraftwellen beträgt im Leerlauf $23.6 \mathrm{kPa}$ und unter Last $80.7 \mathrm{kPa}$. Zur Beurteilung möglicher Biegeschwingungsanregungen der Eigenformen von Stator- und Rotorjoch sind die Anregungsfrequenzen mit den Eigenfrequenzen zu vergleichen. Im Stator führen Drehzahlen $0 \ldots n_{N}$ auf Anregungsfrequenzen der 36. Biegeschwingungsmode zwischen $f_{\min }=0 \mathrm{~Hz}$ bis $f_{\max }=2 \cdot f_{\mathrm{N}}=$ $30.6 \mathrm{~Hz}$. Verglichen mit der nach [14] berechneten Eigenfrequenz

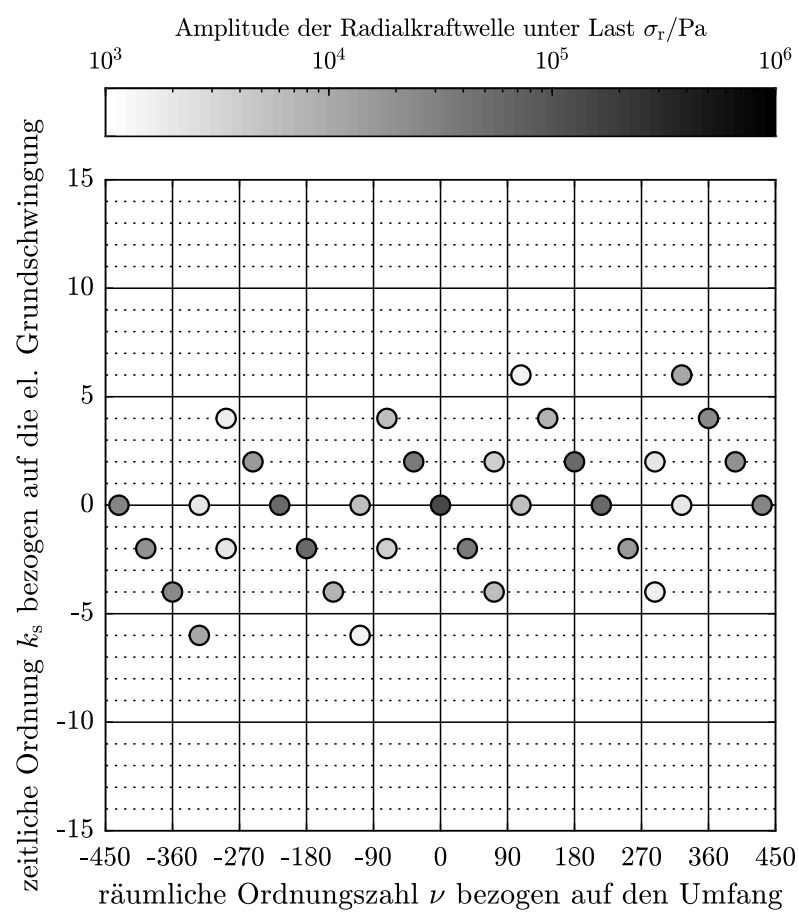

Abb. 21. Maschine Z2, Bemessungslast: Spektrum der errechneten Radialkomponente des Maxwell'schen Zugs längs der Luftspaltmitte

des Statorjochs $f_{\mathrm{s}, \text { eig, } 36}=432.9 \mathrm{~Hz}$ ergibt sich zwischen Anregungsund Eigenfrequenz ein Abstand von mindestens 1.15 Dekaden. Im Rotor führt der Drehzahlbereich $0 \ldots n_{N}$ auf Anregungsfrequenzen der 36. Biegeschwingungsmode zwischen $f_{\min }=0 \mathrm{~Hz}$ bis $f_{\max }=$ $2.4 \cdot f_{\mathrm{N}}=36.7 \mathrm{~Hz}$. Verglichen mit der berechneten Eigenfrequenz des Rotorjochs $f_{r, e i g, 36}=442.6 \mathrm{~Hz}$ ergibt sich zwischen Anregungsund Resonanzfrequenz ein Frequenzabstand von mindestens 1.08 Dekaden. Daher ist eine resonante Anregung der 36. Biegeschwingungsmode für die Maschine Z2 nicht zu erwarten.

\section{Zusammenfassung}

Für die Auslegung und Optimierung eines getriebelosen permanentmagneterregten Außenläufer-Windgenerators mit Zahnspulenwicklung, massivem Rotorjoch und der Möglichkeit zum Weiterbetrieb bei teilweisem Umrichterausfall wurde ein gegliedertes Auswahlverfahren vorgestellt.

Zunächst erfolgte im Hinblick auf die zulässigen Rotorverluste eine schnelle, analytische Berechnung der zu erwartenden Rotorjochwirbelstromverluste. Hierbei zeigte sich, dass nur ZweischichtZahnspulenwicklungen mit geringem Bruchlochnenner in Frage kommen, um die Anzahl und Amplituden zum Rotor asynchron umlaufender Statorfeldwellen zu begrenzen. Die beiden zulässigen Varianten $\mathrm{Z} 1$ und $\mathrm{Z} 2$ mit $q=1 / 2$ und $q=2 / 5$ unterscheiden sich im jeweiligen Verhältnis von Statorstromwärmeverlusten und Rotorjochwirbelstromverlusten. Während die Variante $q=1 / 2$ aufgrund des um 6.7 Prozentpunkte geringeren Wicklungsfaktors höhere Statorstromwärmeverluste aufweist, führt die Variante $q=2 / 5$ aufgrund des ungünstigeren Spektrums zu Rotorjochwirbelstromverlusten, die um den Faktor 8.5 höher sind.

Unter Beibehaltung der analytischen Wirbelstromberechnung wurden im Anschluss die Blechschnitte der beiden Varianten Z1 und Z2 hinsichtlich ihrer Magnetmasse und ihres Wirkungsgrads optimiert. Zur Berechnung der Magnetkreise kamen nichtlineare magnetostatische Finite-Elemente-Simulationen mit MTPA-Suche zum 
Einsatz. Die multikriterielle Optimierung wurde mit Hilfe von genetischen Algorithmen durchgeführt. Die resultierenden Pareto-Fronten zeigen, dass bei moderatem Magnetmasseneinsatz die Variante mit $q=1 / 2$ die Variante mit $q=2 / 5$ hinsichtlich eines höheren Wirkungsgrads dominiert.

Abschließend wurden aus den Pareto-Mengen zwei Maschinen Z1 und Z2 mit einer vorgegebenen Magnetmasse $m_{\text {mag }}=4344 \mathrm{~kg}$ ausgewählt. Für diese Maschinen wurde jeweils eine Zahnspulenwicklung mit Profildrahtwicklung und offenen, ungeschrägten Statornuten ausgelegt. Damit wurden transiente, nichtlineare 2D-FiniteElemente-Simulationen zur Bestimmung des Wirkungsgrads im generatorischen Bemessungspunkt, zum Nachweis der Entmagnetisierfestigkeit bei dreiphasigem Stoßkurzschluss aus Volllast und zur Untersuchung der geräuschanregenden Radialkraftwellen im Hinblick auf die Biegeeigenmoden von Stator- und Rotorjoch durchgeführt. Hierbei zeigte sich, dass die Maschine Z1 $(q=1 / 2)$ gegenüber Z2 $(q=2 / 5)$ einen Wirkungsgradvorteil von 0.2 Prozentpunkten hat, aber wegen der 23-mal höheren Drehmomentwelligkeit und resonanznaher Radialkraftanregungen im Hinblick auf das Geräuschverhalten der Variante mit $q=2 / 5$ unterlegen ist. Allerdings wurden im Rahmen dieser Arbeit keine weiterführenden Maßnahmen zur Reduzierung der Drehmomentwelligkeit erörtert, wie zum Beispiel die Reduzierung von Oberwellen des Erregerfeldes durch entsprechende Konturierung der Oberflächenmagnete oder die aktive Ausregelung der Drehmomentwelligkeit mit Hilfe des Frequenzumrichters.

\section{Danksagung}

Open Access funding provided by Projekt DEAL.

Hinweis des Verlags Der Verlag bleibt in Hinblick auf geografische Zuordnungen und Gebietsbezeichnungen in veröffentlichten Karten und Institutsadressen neutral.

Open Access Dieser Artikel wird unter der Creative Commons Namensnennung 4.0 International Lizenz veröffentlicht, welche die Nutzung, Vervielfältigung, Bearbeitung, Verbreitung und Wiedergabe in jeglichem Medium und Format erlaubt, sofern Sie den/die ursprünglichen Autor(en) und die Quelle ordnungsgemäß nennen, einen Link zur Creative Commons Lizenz beifügen und angeben, ob Änderungen vorgenommen wurden. Die in diesem Artike enthaltenen Bilder und sonstiges Drittmaterial unterliegen ebenfalls der genannten Creative Commons Lizenz, sofern sich aus der Abbildungslegende nichts anderes ergibt. Sofern das betreffende Material nicht unter der genannten Creative Commons Lizenz steht und die betreffende Handlung nicht nach gesetzlichen Vorschriften erlaubt ist, ist für die oben aufgeführten Weiterverwendungen des Materials die Einwilligung des jeweiligen Rechteinhabers einzuholen. Weitere Details zur Lizenz entnehmen Sie bitte der Lizenzinformation auf http://creativecommons.org/licenses/by/4.0/deed.de.

\section{Literatur}

1. Anglada, J. R., Sharkh, S. M., Yuratich, M. A. (2018): Calculation of rotor losses in PM machines with retaining sleeves using transfer matrices. IET Electr. Power Appl., 12(8) 1150-1157.

2. Bianchi, N., Bolognani, S. (1997): Brushless DC motor design: an optimisation procedure based on genetic algorithms. In 1997 VIII international conference on electrica machines and drives (ICEM), Cambridge, UK, September (S. 16-20).

3. Binder, A. (2017): Elektrische Maschinen und Antriebe: Grundlagen, Betriebsverhalten. 2. Aufl. Wiesbaden: Springer.

4. Bolte, E. (2018): Elektrische Maschinen. 2. Aufl. Berlin: Springer.

5. Deb, K. (2001): Multi-objective optimization using evolutionary algorithms. Chiches ter: Wiley.
6. EL-Refaie, A. M. (2010): Fractional-slot concentrated-windings synchronous permanent magnet machines: opportunities and challenges. IEEE Trans. Ind. Electron., 57(1), 107-121.

7. Erd, N., Binder, A. (2018): Eddy currents in solid rotor under spatially intermittent feeding of the stator winding. In 2018 XXIII. International conference on electrical machines (ICEM). Alexandroupolis, Griechenland, September (S. 178-184).

8. Erd, N., Binder, A. (2018): Numerical and analytical analysis of wave harmonics under spatially intermittent feeding. In 2018 XXIII. International conference on electrical machines (ICEM). Alexandroupolis, Griechenland, September (S. 297-303)

9. Fang, Y., Zhang, T. (2018): Vibroacoustic characterization of a permanent magnet synchronous motor powertrain for electric vehicles. IEEE Trans. Energy Convers., 33(1), 272-280.

10. Freeman, E. M. (1968): Travelling waves in induction machines: input impedance and equivalent circuits. Proc. Inst. Electr. Eng., 115(12), 1772-1776.

11. Greig, J., Freeman, E. M. (1967): Travelling-wave problem in electrical machines. Proc. Inst. Electr. Eng., 114(11), 1681-1683.

12. Henke, H. (2011): Elektromagnetische Felder: Theorie und Anwendung. Berlin: Springer.

13. Huth, G. (1995): Nutrastung von permanenterregten AC-Servomotoren mit gestaffelter Rotoranordnung. Electr. Eng., 78(6), 391-397.

14. Jordan, H. (1950): Geräuscharme Elektromotoren. Essen: Girardet.

15. Lawrenson, P. J., Reece, P., Ralph, M. C. (1966): Tooth-ripple losses in solid poles. Proc. Inst. Electr. Eng., 113(4), 657-662.

16. Leitner AG (Sterzing, Italien), Marke Leitwind: Windenergieanlage LTW77. www.leitwind.com/Products/LTW77-1.500-kW. Abruf: 23.05.2020.

17. Meeker, D. C. Finite Element Method Magnetics, Version 4.2 (28Feb2018 Build) http://www.femm.info. Abgerufen am: 12.12.2019.

18. Messoll, A. K. (2014): Untersuchung eines Ringgenerators für Windenergieanlagen der $10 \mathrm{MW}$-Klasse auf Basis statischer Versuchsmodelle und numerischer Simulationen. Universität Kassel, Kassel. Dissertation.

19. Mouty, S. (2013): Conception de machines à aimants permanents à haute densite de couple pour les éoliennes de forte puissance (Entwicklung einer permanentmagneterregten Maschine mit hoher Drehmomentdichte für Windenergieanlagen hoher Leistung). Université de Franche-Comté, Besançon, Frankreich. Dissertation.

20. Polinder, H., Hoeijmakers, M. J., Scuotto, M. (2007): Eddy-current losses in the solid back-iron of PM machines for different concentrated fractional pitch windings. In 2007 IEEE international electric machines drives conference, Antalya, Türkei, Mai (S. 652657).

21. Reichert, K. (2004): PM-motors with concentrated, non overlapping windings, some characteristics. In 2004 XVI international conference on electrical machines (ICEM). Krakau, Polen, September (S. 4).

22. Reichert, K. (2009): Grosse Synchronmaschinen mit Zahnspulen und Permanentmagneterregung, Problemstellungen, Lösungen und Anwendungen. In Internationaler ETG-Kongress 2009, Düsseldorf, Oktober (S. 5).

23. Spinato, F., Tavner, P. J., Bussel, G. J. W. V., Koutoulakos, E. (2009): Reliability of wind turbine subassemblies. IET Renew. Power Gener., 3(4), 387-401.

24. Spooner, E., Williamson, A. C., Catto, G. (1996): Modular design of permanentmagnet generators for wind turbines. IEE Proc., Electr. Power Appl., 143(5), 388-395.

25. Stoll, R. L. (1974): Analysis of eddy currents. Oxford: Clarendon Press.

26. Tessarolo, A. (2017): A survey of state-of-the-art methods to compute rotor eddycurrent losses in synchronous permanent magnet machines. In 2017 IEEE workshop on electrical machines design, control and diagnosis (WEMDCD), Nottingham, UK, April (S. 12-19)

27. The MathWorks Inc.: Find Pareto front of multiple fitness functions using genetic algorithm, https://de.mathworks.com/help/gads/gamultiobj.html. Abruf: 12.12.2019.

28. Ugalde, G., Zhu, Z. Q., Poza, J., Gonzalez, A. (2010): Analysis of rotor eddy current loss in fractional slot permanent magnet machine with solid rotor back-iron. In The XIX international conference on electrical machines (ICEM 2010). Rom, Italien, September (S. 6).

29. Valavi, M., Nysveen, A., Nilssen, R., Lorenz, R. D., Rølvåg, T. (2014): Influence of pole and slot combinations on magnetic forces and vibration in low-speed PM wind generators. IEEE Trans. Magn., 50(5), 11.

30. Štěpina, J. (1987): Matrix analysis of space harmonics of asymmetrical stator windings. IEE Proc., Electr. Power Appl., 134(4), 207-210.

31. Yaramasu, V., Wu, B., Sen, P. C., Kouro, S., Narimani, M. (2015): High-power wind energy conversion systems: state-of-the-art and emerging technologies. Proc. IEEE, 103(5), 740-788.

32. Zhu, Z. Q., Ng, K., Schofield, N., Howe, D. (2004): Improved analytical modelling of rotor eddy current loss in brushless machines equipped with surface-mounted permanent magnets. IEE Proc., Electr. Power Appl., 151(6), 641-650. 
Autoren

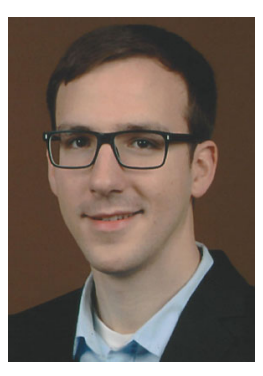

Nicolas Erd

geb. 1989 in Berlin, studierte Elektrotechnik an der TU Berlin und schloss das Studium mit Bachelor (2013) und Master (2015) ab. Seit 2015 ist er wissenschaftlicher Mitarbeiter am Institut für Elektrische Energiewandlung der TU Darmstadt. Seine Forschungsschwerpunkte sind die Auslegung und Berechnung großer permanentmagneterregter Synchronmaschinen für getriebelose Windenergieanlagen.

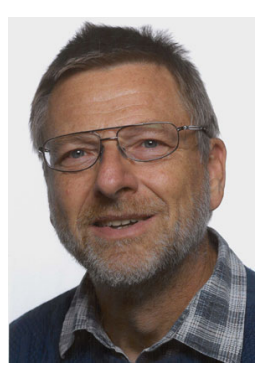

\section{Andreas Binder}

Prof. Dr.-Ing. habil. Dr. h.c. Andreas Binder, Senior Member IEEE, Mitglied VDE, IET, VDI, EPE, studierte bis 1981 Elektrotechnik an der TU Wien (Dipl.-Ing.) und promovierte dort 1988 (Dr. techn.). Von 1981 bis 1983 arbeitete er als Berechnungsingenieur für große Synchrongeneratoren bei der ELIN-Union AG, Wien. Von 1983 bis 1989 war er als wissenschaftlicher Mitarbeiter am Institut für Elek- trische Maschinen und Antriebe, TU Wien, beschäftigt. Von 1989 bis 1997 war er wieder in der Industrie tätig als Gruppenleiter in der Entwicklung von Gleichstrommaschinen und umrichertergespeisten Wechselstrommaschinen bei der Siemens AG, Bad Neustadt, und später in Erlangen. Seit 1994 ist er Lehrbeauftragter mit Habilitation an der TU Wien und gewann 1997 den ETG-Literaturpreis. Seit 1997 leitet er als Professor das Institut für Elektrische Energiewandlung an der TU Darmstadt. Schwerpunkte sind Lehre und Forschung zu elektrischen Maschinen und Antrieben, elektrische Energietechnik, E-Mobilität und Bahnsysteme. Er ist Autor bzw. Co-Autor von über 390 wissenschaftlichen Publikationen, Co-Autor von zwei wissenschaftlichen Büchern, Autor von zwei Büchern über elektrische Maschinen und mehrerer Patente. Seit 2007 ist er Ehrendoktor (Dr. hc.) der technischen Universität Bukarest und seit 2009 Träger der Ehrenmedaille des ETGNDE. Seine aktuellen Forschungsschwerpunkte sind Hochdrehzahlantriebe, permanentmagneterregte Maschinen, Lagerströme, Antriebssysteme für Hybrid- und Elektroautos, Antriebssysteme für Bahnen, Magnetlagerung und Generatorsysteme 